ARTICLE

DOI: $10.1038 / \mathrm{s} 41467-017-02557-2$

\title{
Incomplete prophage tolerance by type III-A CRISPR-Cas systems reduces the fitness of lysogenic hosts
}

Gregory W. Goldberg${ }^{1}$, Elizabeth A. McMillan² ${ }^{2}$ Andrew Varble ${ }^{1}$, Joshua W. Modell ${ }^{1}$, Poulami Samai ${ }^{1}$, Wenyan Jiang ${ }^{1} \&$ Luciano A. Marraffini ${ }^{1}$

CRISPR-Cas systems offer an immune mechanism through which prokaryotic hosts can acquire heritable resistance to genetic parasites, including temperate phages. Cotranscriptional DNA and RNA targeting by type III-A CRISPR-Cas systems restricts temperate phage lytic infections while allowing lysogenic infections to be tolerated under conditions where the prophage targets are transcriptionally repressed. However, long-term consequences of this phenomenon have not been explored. Here we show that maintenance of conditionally tolerant type III-A systems can produce fitness costs within populations of Staphylococcus aureus lysogens. The fitness costs depend on the activity of prophage-internal promoters and type III-A Cas nucleases implicated in targeting, can be more severe in double lysogens, and are alleviated by spacer-target mismatches which do not abrogate immunity during the lytic cycle. These findings suggest that persistence of type III-A systems that target endogenous prophages could be enhanced by spacer-target mismatches, particularly among populations that are prone to polylysogenization.

\footnotetext{
${ }^{1}$ Laboratory of Bacteriology, The Rockefeller University, New York, NY 10065, USA. ${ }^{2}$ Laboratory of Systems Cancer Biology, The Rockefeller University, New York, NY 10065, USA. Andrew Varble and Joshua W. Modell contributed equally to this work. Correspondence and requests for materials should be addressed to L.A.M. (email: marraffini@rockefeller.edu)
} 
rokaryotic organisms can limit the spread of mobile genetic elements (MGEs) with the help of various defense systems, including clustered, regularly interspaced, short palindromic repeat (CRISPR) loci, and CRISPR-associated (cas) genes that function together as adaptive immune systems ${ }^{1,2}$. CRISPR-Cas systems provide immunity to invasive MGEs, such as bacteriophages (phages) ${ }^{3}$ and plasmids ${ }^{4}$, by acquiring short "spacer" sequences from their nucleic acids and incorporating them between repeat sequences of CRISPR locus DNA ${ }^{5,6}$. Transcription of a CRISPR locus, followed by cleavage and processing of its transcripts, generates mature CRISPR RNA (crRNA) guides containing individual spacer sequences ${ }^{7}$. Ultimately, crRNAguided Cas proteins are employed to locate and degrade the nucleic acids of genetic elements that bear a matching target sequence for one or more spacer ${ }^{8,9}$. Four of the six (I-VI) types of CRISPR-Cas systems which have been classified to date ${ }^{8,10}$ include systems that cleave the DNA of their target elements ${ }^{11-14}$.

Given that MGEs can facilitate the spread of beneficial genes within prokaryotic populations, it was proposed that resistance to foreign DNA elements by CRISPR-Cas systems could jeopardize the survival of bacteria which rely heavily on MGE-mediated horizontal gene transfer (HGT) ${ }^{4}$, and in turn promote the evolution of strains that do not harbor CRISPR-Cas systems ${ }^{15,16}$. CRISPR-Cas systems are indeed absent from $\sim 50 \%$ of sequenced bacterial genomes ${ }^{17}$, despite evidence that they can be transferred horizontally ${ }^{18-21}$ and function in heterologous hosts ${ }^{22-24}$. Moreover, the potential for genetic loss of CRISPR-Cas systems has been clearly demonstrated in laboratory settings where both beneficial plasmids and temperate phages were targeted. During the anti-plasmid CRISPR-Cas immune response, population bottlenecks imposed by antibiotics were found to select for mutant or deleted CRISPR-Cas systems when a target plasmid carrying resistance to the antibiotic was introduced prior to treatment ${ }^{25-27}$. Similar outcomes were observed when type I and type II CRISPR-Cas systems were engineered to target temperate phages that produce antibiotic-resistant lysogens ${ }^{28,29}$. In this manner, indiscriminate targeting of beneficial elements might impede the distribution of CRISPR-Cas systems in natural populations.

Fitness costs associated with the upkeep of CRISPR-Cas systems, including costs which result from basal expression and the occasional acquisition of toxic spacers that target their host's chromosome, have also been proposed to influence the distribution of these systems $s^{2,30-32}$. In certain cases, toxic chromosomal targeting may result from CRISPR-Cas immunity directed at temperate phages which integrate into the host chromosome as prophage DNA during lysogenic infections ${ }^{28,29}$. We previously demonstrated that the type III-A system from Staphylococcus epidermidis RP62 $\mathrm{a}^{33}$ can avert targeting of integrated lambda-like (lambdoid) prophages and give rise to "conditionally tolerant" lysogens in which the CRISPR-Cas system and prophage target have not been genetically altered ${ }^{29}$. In other words, we observed a stable co-existence between functional type III CRISPR-Cas systems and prophage target DNA in lysogenic hosts. Type III systems are distinct from other DNA-cleaving CRISPR-Cas systems where targeting occurs only at sequences which are transcribed, and requires crRNAs which are complementary to the nascent transcripts ${ }^{29,34}$. The type III-A system of S. epidermidis RP62a was further shown to encode a targeting complex with nucleases that license cleavage of RNA in addition to $\mathrm{DNA}^{13}$, as well as an auxiliary RNase (Csm6) that can assist in the degradation of phage transcripts during lytic infection ${ }^{35}$. Collectively, these type III-A nucleases allow the system to prevent temperate phage propagation when its targets are transcribed during lytic infection or prophage induction, and yet tolerate prophages while their targets are sufficiently repressed in the chromosome.
However, type-III A spacers displaying a perfect match with an endogenous prophage are yet to be found in the completed staphylococcal genomes. Thus, it remains to be determined whether conditional tolerance can influence the long-term persistence of type III-A systems in natural populations of lysogenic Staphylococci, including $S$. aureus lineages that are polylysogenized with more than one prophage.

In this work, we use laboratory derivatives of a model clinical isolate, $S$. aureus Newman ${ }^{36}$, and show that maintenance of conditionally tolerant type III-A systems can become a fitness liability for lysogenic hosts. We also provide evidence that the costs result from transcription-dependent targeting at prophage loci, and investigate the effects of different spacers within populations of single or double lysogens.

\section{Results}

Incomplete prophage tolerance by type III-A systems. In a previous work ${ }^{29}$, the CRISPR-Cas system of S. epidermidis RP62a was re-engineered to contain spacers targeting the temperate phages of $S$. aureus Newman, and introduced into heterologous RN4220 37 or TB4 hosts ${ }^{38}$ on pC194-based plasmids ${ }^{39}$. TB4 is a prophage-cured derivative of Newman that can be re-infected with the native phages, including $\Phi$ NM1 and $\Phi$ NM4. One of our previously tested spacer sequences was modeled from a spacer occurring naturally in the type III-A system of $S$. argenteus MSHR1132 ${ }^{40}$, and was found to license conditional tolerance regardless of its five mismatches (Supplementary Fig. 1a) relative to $\Phi$ NM1 phage DNA target sequences ${ }^{29}$. When a type III-A plasmid programmed with a perfectly matching variant of this spacer $\left(g p 32^{\star}\right)$ was introduced into TB4:: $\Phi$ NM1 lysogens, a small colony phenotype was clearly apparent when compared with isogenic lysogens containing the gp32 or non-targeting CRISPR-Cas plasmids (Supplementary Fig. 1b). We hypothesized that this phenotype reflects a general growth rate reduction relative to non-targeting lysogens, and sought to quantify it more precisely in terms of a spacer-dependent fitness cost. This was accomplished by marking the TB4:: $\Phi$ NM1 lysogens with small plasmids ${ }^{41,42}$ conferring resistance to either tetracycline (Tet) or erythromycin (Erm), and measuring their relative abundances over time in pair-wise competition co-cultures. As anticipated from the differences in colony size, lysogens with the perfectly matching $g p 32^{*}$ spacer seemed to decline in relative abundance more rapidly than lysogens with the mismatched spacer, when each were competed against the non-targeting lysogen (Supplementary Fig. 1c, d). Supplementary Table 1 lists average selection coefficients ( $s$, where $s<0$ and $s>0$ imply that the targeting lysogens are at a fitness advantage or fitness disadvantage, respectively), calculated for each competition experiment performed in this study. We measured higher selection coefficients with the perfectly matching $g p 32^{*}$, but this difference was not statistically significant (Supplementary Table 2; linear mixed effect model (lme), $p=0.0983)$. A summary of the statistical tests employed in this study is provided in Supplementary Table 2. To probe the generality of spacer-dependent fitness costs, we tested three additional spacers with perfect identity to sequences within $g p 5$, $g p 16$, and $g p 43$ of $\Phi$ NM1 (Fig. 1a), and assayed them for relative fitness using the same setup. Significant fitness costs were measured for each of the conditionally tolerant lysogens when their selection coefficients were compared with those of control cocultures (Fig. 1b-d; lme, $p<0.05$ ), in which both competing lysogens harbored the same non-targeting spacers and no significant changes in relative abundance were observed over time (Fig. 1e; one-way ANOVA, $p=0.6342$ ).

Previous studies have demonstrated that chromosomal targeting by CRISPR-Cas systems in bacteria is detrimental to growth 
a $\begin{array}{llll}\text { gp5 } & \text { gp16 } & \text { gp32 }\end{array}$

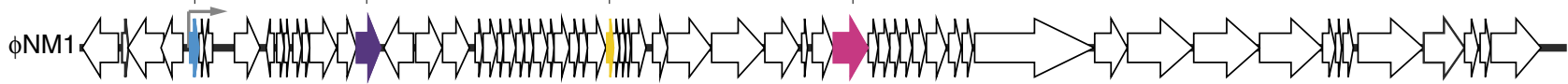

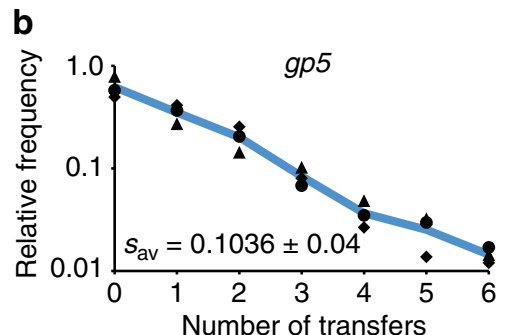

C

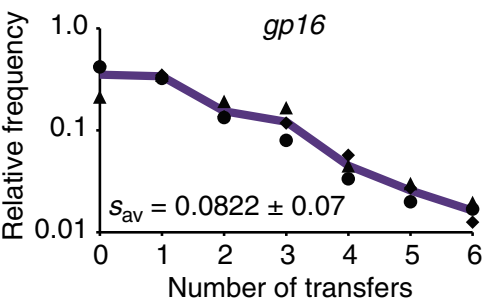

d
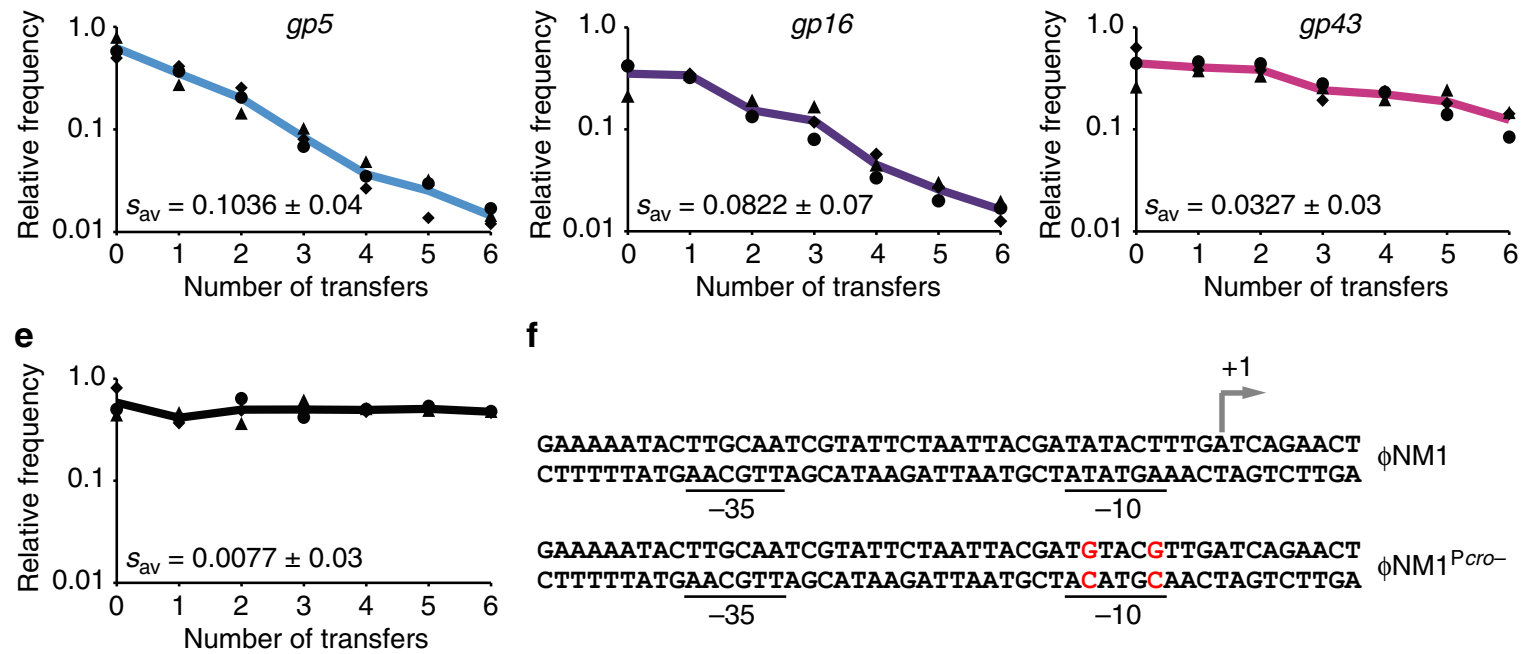

g

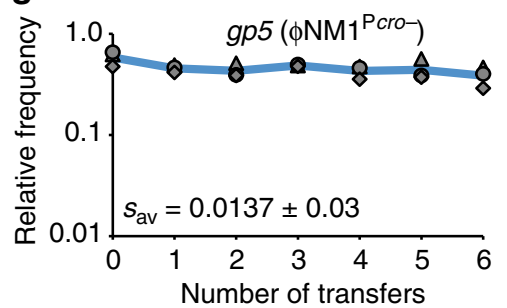

$\mathbf{f}$

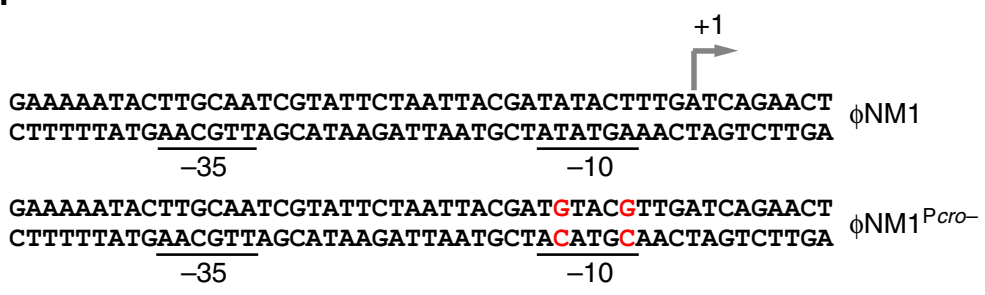

h

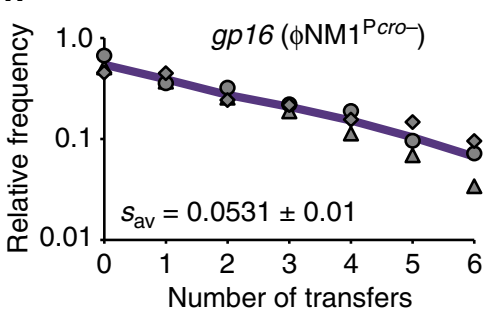

i

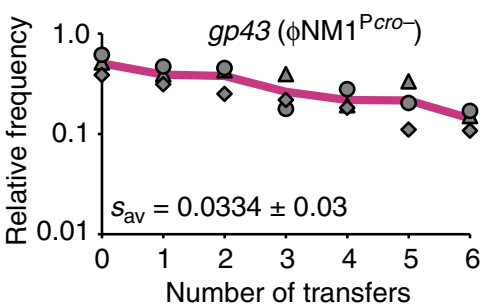

Fig. 1 Incomplete prophage tolerance by type III-A systems can be licensed by prophage-internal promoter activity. a Schematic representation of the integrated $\Phi$ NM1 prophage genome. The $g p 5, g p 16, g p 32$, and gp43 open reading frames are colored to denote inclusion of a target sequence for one or more spacers tested in this work. Gray bent arrow indicates the position of $\Phi$ NM1's early lytic promoter $\left(P_{\text {cro }}\right)$. b-d Pairwise competition experiments for three perfectly matching spacers that license conditional tolerance of $\Phi \mathrm{NM1}$. In each case, an Erm ${ }^{\mathrm{R}}$-marked control lysogen harboring the parent vector with non-targeting spacers was competed against a Tet ${ }^{R}$-marked conditionally tolerant lysogen. Relative frequencies ( $y$-axis) are plotted against the number of transfers ( $x$-axis), with one transfer per day. Individual values from each biological replicate $(n=3)$ are depicted in black as a triangle, circle, or rhombus. Solid lines represent the average change in relative frequency across the three replicates, and share color coding with the target genes outlined in a. e Control co-culture experiment. Pairwise competition was performed as in b-d above, except that differentially marked TB4:: $\Phi$ NM1 lysogens both harbor the same parent vector with non-targeting spacers. $\mathbf{f}$ Sequences of the wild-type $\Phi N M 1$ early lytic promoter and its inactive $\Phi N M 1$ Pcro- variant with point mutations (red lettering) in the -10 element. The location of this promoter, along with the approximate position of its transcriptional start site (+1), was inferred from RNA-sequencing analysis of $\Phi$ NM1 during lytic infections ${ }^{29}$. This designation is further supported by research on S. aureus lambdoid phages with related regulatory architecture ${ }^{77-79} \cdot \mathbf{g}$-i Pairwise competition experiment as in $\mathbf{b}$-d, except that targeting and control lysogens both contain the $\Phi N M 1^{P c r o-}$ prophage mutations. All $s_{\text {av }}$ values are derived from Supplementary Table 1 and provide mean \pm standard deviation (s.d.)

and potentially lethal ${ }^{27,30}$, even in cases where prophage loci are targeted $^{28,29,43}$. Type III-A systems can tolerate intact chromosomal targets, but only while they are sufficiently repressed. In light of these findings, we hypothesized that the spacer-dependent fitness costs could be derived from low levels of type III-A chromosomal targeting, licensed by transcription at prophage loci. Transcription of prophage loci can occur in a subset of cells during spontaneous prophage induction ${ }^{44}$. However, spontaneous prophage induction is lethal in the absence of targeting spacers, and it is therefore unexpected that non-targeting lysogens would be more fit than lysogens with targeting spacers, as we observed in our competition experiments (Fig. 1). Furthermore, we determined that all of these targeting spacers can disrupt phage lytic propagation via spontaneous (Supplementary Fig. 2a) or MMC-stimulated (Supplementary Fig. 2b) prophage induction. Therefore, if the fitness costs were derived from targeting effects which occur during prophage induction, each spacer might be expected to produce similar fitness costs. Instead, we observed significantly different fitness costs for at least one of the spacers we tested across six transfers (Fig. 1b-d; one-way ANOVA, $p=$ 0.0499 ). To rule out the possibility that prophage induction is required for the fitness defects observed in competition assays, we disrupted $\Phi N M 1$ 's inducibility by introducing a serine to alanine (S124A) missense mutation in the catalytic domain of its $c I$-like repressor, generating $\Phi N M 1^{\text {ind }}{ }^{-}$(Supplementary Fig. 2c). The CI-like repressors of lambdoid phages possess a serine protease activity that is required for prophage induction via $\operatorname{Rec}^{*}$ stimulated autoproteolysis and de-repression ${ }^{45-47}$. This mutation disrupted both spontaneous (Supplementary Fig. 2a) and MMCtriggered (Supplementary Fig. 2d) prophage induction. Although we were unable to isolate stable transductants of TB4:: $\Phi \mathrm{NM}^{\text {ind }}{ }^{\text {- }}$ harboring the $g p 5$-targeting plasmid, fitness costs associated with 
each of the $g p 16$ - and $g p 43$-targeting plasmids were not significantly reduced in this prophage-mutant background (Supplementary Fig. 2e-,f; lme, $p=0.9786$ and $p=0.5431$, respectively), when selection coefficients were compared to those obtained in the wild-type background (Fig. 1c, d).

The results above confirm that de-repression during prophage induction is not required for fitness costs associated with conditionally tolerant type III-A systems in lysogens. We therefore postulated that the costs could be licensed by unregulated transcription from one or more promoter within the target prophage. To explore this possibility, we introduced inactivating mutations into the rightward promoter of $\Phi$ NM1 (Fig. 1a, f) that is predicted to be involved in the lysis/lysogeny decision of lambdoid phages ${ }^{48}$, generating $\Phi \mathrm{NM}^{\mathrm{P} c r o-}{ }^{-}$. As expected, mutation of this promoter was sufficient to abolish the prophage's lytic cycle in two assays (Supplementary Figs. 2a and g), so we proceeded to test these mutant lysogens in competition co-cultures. The $\mathrm{P}_{\text {cro }}$ mutation virtually abolished the fitness cost associated with the $g p 5$ spacer, which targets a sequence immediately downstream of the promoter, such that selection coefficients were no longer significantly higher than those obtained in control competitions (Fig. 1g; lme, $p=0.2721$ ). In contrast, the costs associated with the $g p 16$ and $g p 43$ spacers were only partially reduced or not significantly reduced at all, respectively, when compared to their costs in the wild-type $\Phi$ NM1 background (Fig. 1h, i; lme, $p=0.0321$ and $p=0.6711$, respectively). Potentially, these fitness costs are licensed by leaky transcription from other sequences in the prophage genome. Taken together, these results demonstrate that conditionally tolerant type III-A systems can impose spacer-dependent fitness costs even if their target prophages are not inducible, and rather suggest that the costs arise from leaky transcription of prophage targets within otherwise stably lysogenic cells.

Contributions of targeting nucleases to fitness costs. Having demonstrated that lysogenic hosts which maintain type III-A systems can incur spacer-dependent fitness costs, we wanted to determine whether this phenomenon results from CRISPR-Cas targeting. To this end, we measured fitness costs associated with the gp16 spacer in the presence of mutations in Cas nuclease active sites previously implicated in targeting by the S. epidermidis type III-A system (Fig. 2a). Mutation of Csm3 (D32A) was previously shown to abolish crRNA-guided transcript cleavage, while mutation of Cas10's palm polymerase domain (D586A, D587A) was shown to abolish the complex's capacity for transcriptiondependent cleavage of $\mathrm{DNA}^{13}$. In addition, mutations in the HEPN domain of the Csm6 RNase (R364A, H369A) were shown to abolish its transcript degradation activity and eliminate its contributions to phage defense $\mathrm{e}^{35}$. Whereas no significant fitness cost reduction was detected with the csm3 mutant when compared to the wild-type plasmid harboring $g p 16$ (Fig. $2 \mathrm{~b}$; lme, $p=$ 0.5655 ), fitness costs appeared to be completely abolished with the cas 10 palm domain mutant (Fig. 2c), given that no significant difference was observed when selection coefficients were compared with that of control competitions (lme, $p=0.5341$ ). Notably, both the $\operatorname{csm} 6$ and $\operatorname{csm} 3 / \operatorname{csm} 6$ mutants also displayed a significantly reduced fitness cost, compared to the wild-type $g p 16$ plasmid (Fig. 2d, e; lme, $p=0.0026$ and $p=0.0272$, respectively). These findings imply that the targeting activities of Cas10 and Csm6 contribute to growth defects associated with incomplete tolerance of prophages by type III-A CRISPR-Cas systems.

Consequences of conditional targeting in polylysogens. Clinical isolates of $S$. aureus are seldom found to possess CRISPR-Cas systems ${ }^{49}$, and in many cases are polylysogens harboring more than one prophage $\mathrm{e}^{50,51}$. The clinical isolate $S$. aureus Newman, for example, does not harbor an endogenous CRISPR-Cas system but harbors four heteroimmune lambdoid prophages ${ }^{38}$. However, among the staphylococcal type III-A CRISPR-Cas systems identified so far some spacers have been found to match conserved temperate phage lytic genes ${ }^{29,49}$, and presumably offer the potential to target multiple phages. These observations prompted us to investigate the consequences of maintaining type III-A systems in a polylysogenic scenario where more than one prophage can be targeted. This was accomplished using $\Phi$ NM1 and $\Phi N M 4$, which integrate into distinct chromosomal loci but share extensive regions of sequence homology, including a perfectly conserved target for the gp16 spacer (Fig. 3a). Provided that each target sequence falls within lytic gene clusters which are sufficiently repressed during lysogeny, we anticipated that polylysogeny would be tolerated even in the presence of perfectly matching spacers. To confirm this, TB4:: $\Phi$ NM4 lysogens harboring CRISPR-Cas plasmids with spacers matching to $\Phi$ NM1's $g p 5, g p 16$, or $g p 43$ targets were infected with an erythromycin resistance-conferring derivative of $\Phi \mathrm{NM} 1\left(\Phi \mathrm{NM} 1-\mathrm{Erm}^{\mathrm{R} 2}\right)$ that allows quantification of newly formed lysogens. When compared with control lysogens harboring a fully mismatched $(35 \mathrm{~mm})$ spacer, we found no significant differences in the frequency of $\Phi N M 1-E r{ }^{R} 2$ secondary lysogenization for lysogens with the singly ( $g p 5, g p 43)$ and doubly ( $g p 16)$ targeting spacers (Fig. 3b; one-way ANOVA, $p=0.1906$ ), while plaque formation on the same hosts was strongly reduced in the presence of targeting spacers (Fig. 3c). However, differences in colony size phenotype were clearly discernible among the resulting double lysogens. Relative to double lysogens with the control spacer, double lysogens with the $g p 5$ or gp43 spacers which do not target $\Phi$ NM4 had a less severe colony size reduction than lysogens with the gp16 spacer targeting both prophages (Fig. 3d). To determine whether these phenotypes reflect discrepancies in relative fitness, we mixed unmarked double lysogens harboring each of the three spacers into a single batch and competed them in liquid culture for 3 days. At each transfer interval, spacer abundances were measured by deep sequencing, and the frequency of each targeting spacer was calculated relative to the control spacer's frequency in the sample. These pairwise relative frequencies were then used to calculate selection coefficients for each strain (Supplementary Table 1). Our analysis indicates that the fitness cost associated with the dual-targeting gp16 spacer in double lysogens was significantly greater than that obtained with the $g p 5$ and $g p 43$ spacers targeting only $\Phi$ NM1 (Fig. 3e; one-sided $t$ tests, $p=0.0095$ and $p=0.0049$, respectively). Importantly, although the gp16-associated cost in double lysogens was significantly higher than its average cost in pairwise competitions with either of the marked TB4::ФNM1 (Fig. 1c; one-sided $t$ test, $p=0.0056$ ) or TB4:: $\Phi N M 4$ single lysogens (Supplementary Fig. 3; one-sided $t$ test, $p$ $=0.0095)$ across the first three transfers, we did not observe a significant difference when comparing the $g p 5$ - or $g p 43$-associated costs in double lysogens (Fig. 3e) to their average costs in pairwise competitions with marked TB4:: $\Phi$ NM1 single lysogens across the first three transfers (Fig. 1b, d; two-sided $t$ tests, $p=0.6322$ and $p$ $=0.5643$ ). These results demonstrate that type III-A CRISPR-Cas systems can become a greater fitness liability if they target multiple temperate phages, despite their capacity for conditional tolerance.

Effects of spacer-target mismatches on fitness costs. The above results confirm that type III-A systems that target multiple temperate phages are not a barrier to polylysogenization per se, even if they possess perfectly matching spacers. However, when searching the fully sequenced staphylococcal genomes which were 
a

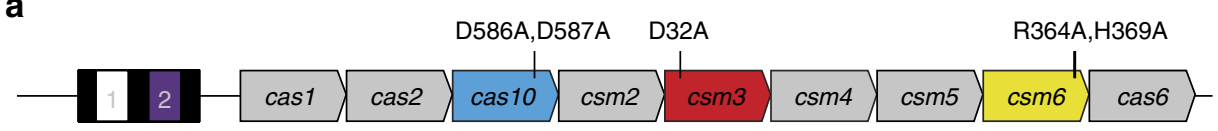

\section{b}
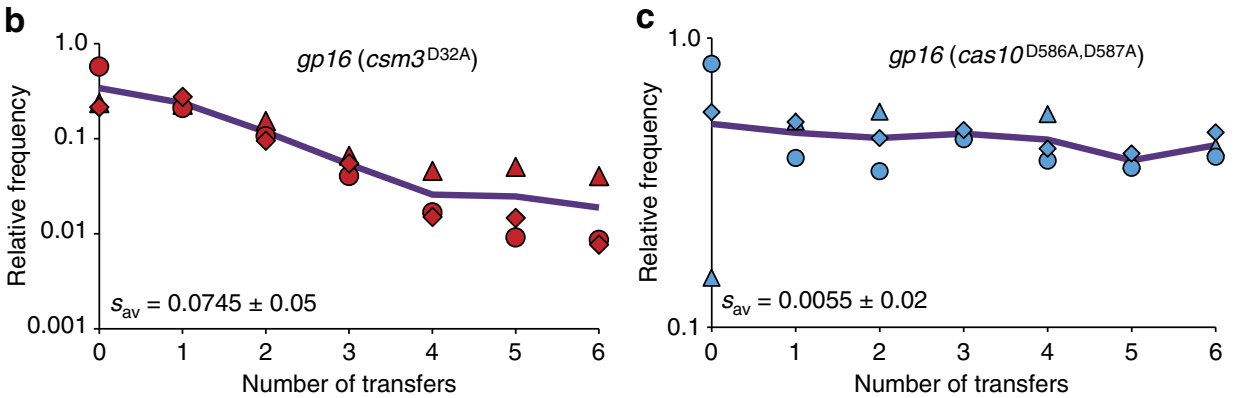

d

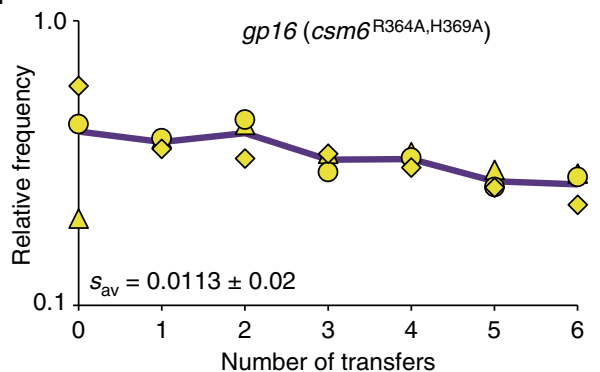

Fig. 2 Effect of targeting nuclease active site mutations on fitness costs associated with type III-A systems. a Schematic diagram summarizing the arrangement of CRISPR-Cas loci used throughout the work, with position 2 of the CRISPR array occupied by the gp16 spacer (purple rectangle) in this example. The naturally occurring nes spacer (white rectangle) from S. epidermidis RP62a is maintained at position 1 in all cases. Open reading frames (ORFs) encoding Cas nucleases implicated in targeting are highlighted in blue (cas10), red (csm3), or yellow (csm6), and the positions of their active site mutations are labeled to scale within each ORF. Other features of the diagram are not drawn to scale. b-e Pairwise competition experiments for mutant CRISPR-Cas plasmids harboring the gp16 spacer with perfect matches to $\Phi$ NM1. In each case, an Erm ${ }^{R}$-marked control lysogen harboring the wild-type parent vector with non-targeting spacers was competed against a Tet ${ }^{R}$-marked lysogen harboring a mutant CRISPR-Cas plasmid with the gp16 spacer. Relative frequencies ( $y$-axis) are plotted against the number of transfers ( $x$-axis), with one transfer per day. Individual values from each biological replicate $(n=3)$ are depicted as a triangle, circle, or rhombus, and are colored to match the ORFs highlighted in a for each mutant, or orange for the csm3/csm6 double mutant. Solid lines represent the average change in relative frequency across the three replicates, and are colored purple to match the gp 16 spacer's target gene as outlined in Fig. 1. The $s_{a v}$ values are derived from Supplementary Table 1 and provide mean \pm s.d

previously found to contain type III-A systems ${ }^{49}$, we were unable to identify cases where perfectly matching targets co-existed with spacers within the same genome. Meanwhile, as previously reported, one of these genomes possesses a spacer with two partially mismatched targets (each within a separate prophage), and we were able to confirm this using modified search parameters. Having found, also, that our partially mismatched $g p 32$ spacer is still capable of targeting $\Phi \mathrm{NM} 1$ during the lytic cycle (Supplementary Fig. 2) but displayed a fitness cost which appeared to be lower (although this reduction was not statistically significant) than that of its perfectly matched $g p 32^{\star}$ variant in single lysogens (Supplementary Fig. 1), we wondered if spacertarget mismatches could likewise help to minimize fitness tradeoffs in polylysogenic populations. To investigate this, we constructed a series of mismatched spacers based around our $g p 16$ spacer, which targets an identical sequence found in both $\Phi$ NM1 and $\Phi$ NM4 (Fig. 3a). It was previously shown that mismatches most readily abrogate type III targeting when they are distributed at both the $5^{\prime}$ and $3^{\prime}$ ends of the spacer ${ }^{49,52}$. Accordingly, we introduced increasing numbers of mutations at both ends of the gp16 spacer (Fig. 4a) in an effort to find a minimal number of mismatches that reduces fitness costs without eliminating targeting during the lytic cycle. The resulting CRISPR-Cas plasmids were then transduced into unmarked TB4:: $\Phi$ NM4+ $\Phi$ NM1 double lysogens, as well as unmarked TB4::ФNM1 single lysogens for comparison. We first examined the potential of each spacer to disrupt the phage lytic cycle in single and double lysogens during MMC-stimulated prophage induction. In both cases, the number of plaque-forming units recovered after induction was reduced to a similar degree by the spacers with six or fewer mismatches, while targeting was undetectable with eight or more mismatches (Fig. $4 \mathrm{~b}$ ). The gp16 variant with six mismatches was also confirmed to be protective in lytic infection assays with $\phi N M 1 \gamma 6^{29}$, a virulent mutant of $\$ \mathrm{NM} 1$ (Supplementary Fig. 4a, b). In particular, when comparing the average fold change in relative abundance before and after infection of competition co-cultures (Supplementary Fig. 4c), we observed no significant difference for the isogenic strains with zero- and six-mismatched spacers $(0.5982 \pm 0.09$ and $0.7842 \pm 0.02$, respectively; two-sided $t$ test, $p$ $=0.0669$ ). Evidently, survival was not impaired by the presence of even six spacer-target mismatches during the course of the experiment. These results are well in line with previous reports demonstrating that our type III-A system's in vivo targeting requirements depend on such factors as mismatch position, sequence context, and assay conditions; in some cases even ten spacer-target mismatches can be introduced before targeting of transcribed DNA elements is abrogated, while in other cases as few as three are sufficient to abrogate targeting $29,35,53,54$.

We next tested each group of single or double lysogens in batch competition experiments. Again, spacer abundances were measured by deep sequencing at each transfer interval for 3 days, and the frequency of each spacer was calculated relative to the fully 
a

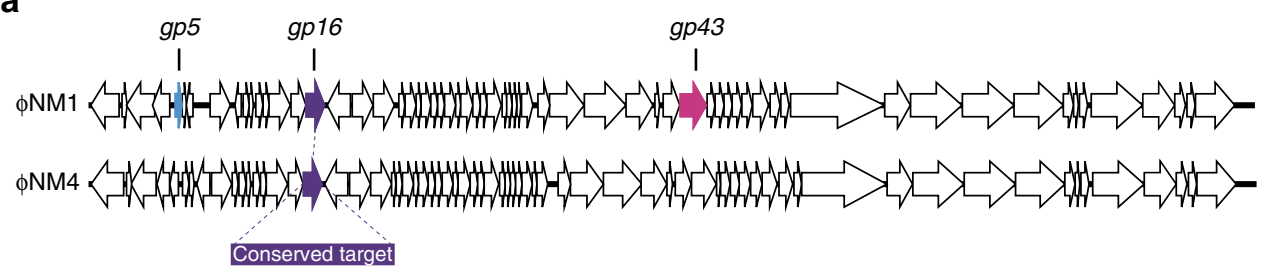

b

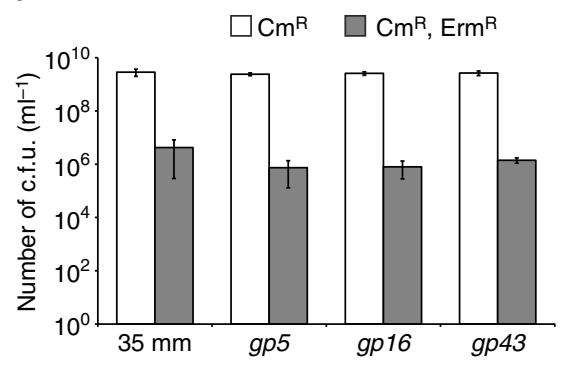

d

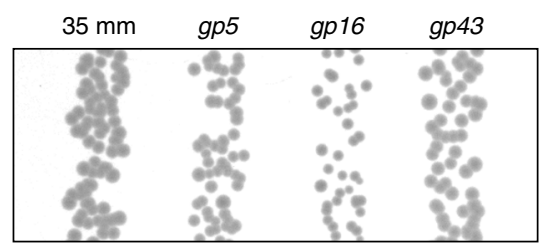

C

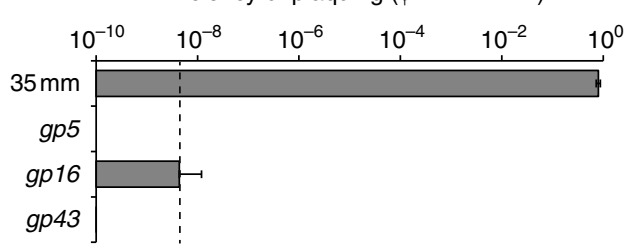

e

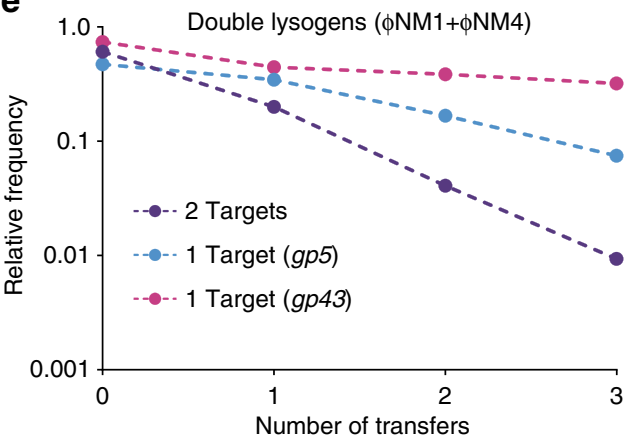

Fig. 3 Type III-A systems which target multiple temperate phages can impose greater fitness costs in polylysogenic hosts. a Schematic representation of the integrated $\Phi$ NM1 and $\Phi$ NM4 prophage genomes. Colored open reading frames (ORFs) denote the presence of a target sequence for perfectly matching spacers. $\Phi N M 1$ 's gp16 sequence includes a target that is found with $100 \%$ conservation in gp 17 of $\Phi N M 4$, as indicated by color-matched ORFs (purple). b Secondary lysogenization of TB4:: $\Phi$ NM4 with $\Phi N M 1-E r m^{R 2}$ in the presence of the $35 \mathrm{~mm}$, gp5, gp16, or gp43 CRISPR-Cas plasmids as indicated. The "35 mm" spacer is a fully mismatched variant of the gp16 spacer that serves as a non-targeting control. Lysogenization was quantified as the concentration of $\mathrm{Cm}^{\mathrm{R}}+\mathrm{Erm}^{\mathrm{R}}$ colony-forming units (colony-forming units per milliliter) in each culture following treatment with $\Phi N M 1-E r m^{R 2}$. For comparison, total recipients were quantified as the concentration of $\mathrm{Cm}^{\mathrm{R}}$ colony-forming units in each untreated culture. Error bars, mean $\pm \mathrm{s} . \mathrm{d}$. ( $n=3$, biological replicates). c Plaquing efficiency of $\Phi$ NM1-Erm ${ }^{R 2}$ on TB4:: $\Phi$ NM4 lysogens harboring the different CRISPR-Cas plasmids tested in $\mathbf{b}$, as indicated by the labels. Efficiency ratios were calculated for each plasmid relative to plaques quantified on a TB4 lawn harboring the non-targeting parent vector. Dotted line represents the limit of detection under these assay conditions. Error bars, mean \pm s.d. ( $n=3$, technical replicates). d Colony size comparison of TB4:: $\Phi$ NM4+ $\Phi N M 1-E r{ }^{R 2}$ double lysogens harboring the different CRISPR-Cas plasmids tested in $\mathbf{b}$, as indicated by the labels above. Picture is representative of a biological replicate for each strain plated in the presence of chloramphenicol to maintain CRISPR-Cas plasmids. e Batch competition experiment with unmarked TB4:: $\Phi N M 4+\Phi N M 1$ double lysogens harboring the different CRISPR-Cas plasmids tested in $\mathbf{b}$. As outlined in the methods, deep sequencing was used to determine targeting spacer frequencies relative to the $35 \mathrm{~mm}$ non-targeting spacer within the sample ( $y$-axis), and plotted against the number of transfers ( $x$-axis, one transfer per day). Dashed lines illustrate the change in relative frequency across time points, and are color-coded for their targets according to the schematic in a

mismatched control spacer's frequency in the sample. Our analysis indicates that the fitness costs were significantly reduced for spacers with four or six mismatches when compared to those of the perfectly matching gp16 spacer within their respective populations of single (Fig. $4 \mathrm{c}$; one-sided $t$ tests, $p=0.0280$ and $p=$ 0.0290 , respectively) or double (Fig. $4 \mathrm{~d}$; one-sided $t$ tests, $p=$ 0.0436 and $p=0.0281$, respectively) lysogens. As expected from the observed lack of targeting during the phage's lytic cycle, no significant costs were measured for any of the spacers with eight or more mismatches in either population (one-way ANOVAs, $p$ $=0.7408$ and $p=0.6265$, respectively), when selection coefficients were compared with that of the average obtained in control TB4:: $\Phi N M 1$ pairwise competitions across their first three transfers (Fig. 1e). Importantly, we observed no significant difference between the cost associated with the perfectly matching gp16 spacer in competitions with marked and unmarked TB4:: $\Phi N M 1$ single lysogens (Fig. $1 \mathrm{c}$ and 4c; two-sided $t$ test, $p=$ 0.3862 ), and this served to validate our usage of deep sequencing to assess relative fitness. We also found that the cost associated with the perfectly matching $g p 5$ spacer targeting only $\Phi$ NM1 (Fig. 3e) was not significantly different from the costs measured for the dual-targeting spacers in double lysogens possessing either four or six mismatches (Fig. $4 \mathrm{~d}$; two-sided $t$ tests, $p=0.4508$ and $p=0.1433$, respectively). Altogether, these results confirm that spacer-target mismatches can alleviate fitness costs associated with incomplete prophage tolerance by type III-A systems in (poly)lysogenic staphylococci, even in cases where immunity to lytic infections is not abrogated.

\section{Discussion}

We report here that certain conditionally tolerant type III-A CRISPR-Cas systems can put their (poly)lysogenic hosts at a fitness disadvantage in mixed populations of $S$. aureus, despite the potential for these CRISPR-Cas systems and their prophage target(s) to co-exist in (poly)lysogenized clones. The fitness costs varied in severity with the position of targets within the prophage genome, and likely result from incomplete prophage tolerance by 
a

5' GCTCCACTGATATACTCAGTCGGGAGGACGCCAAG $3^{\prime}$

5' TAGAACAGTCGCGCAGACTGATTTCTTCATAACCT $3^{\prime}$

5' TCGAACAGTCGCGCAGACTGATTTCTTCATAACAT 3'

5' TCGCACAGTCGCGCAGACTGATTTCTTCATACCAT 3'

5' TCGCAAAGTCGCGCAGACTGATTTCTTCAGACCAT 3'

5' TCGCAAATTCGCGCAGACTGATTTCTTAAGACCAT 3'

5' TCGCAAATTAGCGCAGACTGATTTCGTAAGACCAT $3^{\prime}$

5' TCGCAAATTAGAGCAGACTGATTGCGTAAGACCAT 3 '

5' TCGCAAATTAGAGAAGACTGAGTGCGTAAGACCAT 3 '

5' TCGCAAATTAGAGAATACTTAGTGCGTAAGACCAT 3 '

C

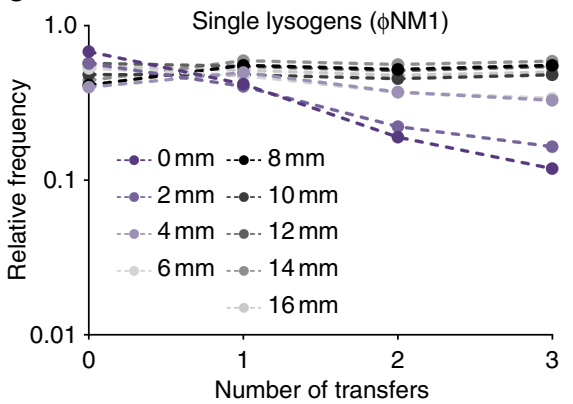

b

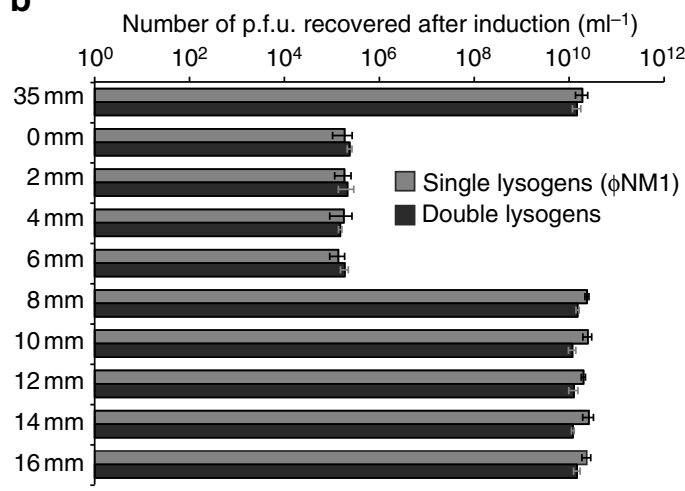

d

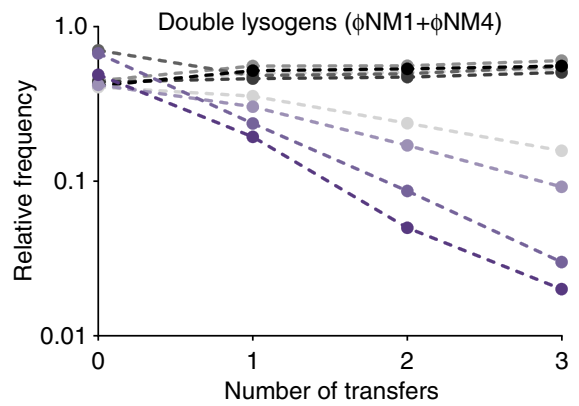

Fig. 4 Effects of spacer-target mismatches in single and double lysogens harboring a CRISPR-Cas system with dual-targeting potential. a Sequence summary of the gp16 spacer and its mismatched variants. Black lettering indicates sequences found in the parental gp16 spacer; red lettering indicates mutated base pairs. A subset of transversion mutations were used to avoid introducing $\mathrm{G}: \mathrm{U}$ pairing when complementarity between the crRNA and transcribed target RNA is considered. $\mathbf{b}$ Quantification of plaque forming unit concentrations (plaque-forming units per milliliter) in filtered supernatants from subcultures of single (TB4:: $\Phi$ NM1) or double (TB4:: $\Phi$ NM4+ $\Phi N M 1)$ lysogens harboring the spacers depicted in a, recovered $4 \mathrm{~h}$ post treatment with Mitomycin $C$ at $1.0 \mu \mathrm{g} \mathrm{ml}^{-1}$. Error bars, mean \pm s.d. $(n=3$, biological replicates). c Batch competition experiment with unmarked TB4:: $\Phi$ NM1 single lysogens harboring CRISPR-Cas plasmids with the perfectly matched gp16 spacer or one of several mismatched derivatives shown in a. As outlined in the methods, deep sequencing was used to determine targeting spacer frequencies relative to the $35 \mathrm{~mm}$ spacer within the sample ( $y$-axis), and plotted against the number of transfers ( $x$-axis, one transfer per day). Dashed lines illustrate the change in relative frequency across time points, and are color-coded according to the labeled inset. $\mathbf{d}$ Same as in $\mathbf{c}$, except that unmarked TB4:: $\Phi N M 4+\Phi N M 1$ double lysogens were used in the experiment

the system's transcription-dependent targeting machinery. We previously showed that transcription-dependent chromosomal targeting by the type III-A system of S. epidermidis RP62a causes severe growth defects ${ }^{29,35}$, and similar effects have been observed with other types of CRISPR-Cas systems ${ }^{27,28,30}$. At least in part, this appears to result from toxicity associated with frequent nicking or cleavage of the host's chromosomal DNA. Minor growth defects, in line with the fitness costs described in this work, might instead be expected if type III-A chromosomal targeting were infrequent; e.g., licensed by transcription in a small subpopulation of cells. When lambdoid lysogens are cultured under standard growth conditions, transcription from lytic promoters can occur in a subpopulation of cells undergoing the lytic cycle via spontaneous prophage induction ${ }^{44}$. However, our results suggest that the fitness costs might depend instead on leaky transcription of prophage targets occurring within stably lysogenic cells, rather than transcription occurring in a subpopulation of cells that is undergoing spontaneous prophage induction (Supplementary Fig. 2). It is also important to note that due to the use of a high copy plasmid-based type III-A CRISPR-Cas system, the fitness costs we observed may be exacerbated due to a higher expression of this system. Consistent with a requirement for transcription at prophage target loci, costs associated with the $g p 5$ spacer were virtually undetectable when the $\mathrm{P}_{\text {cro }}$ promoter immediately upstream of its target was inactivated (Fig. 1g). In contrast, mutation of $\mathrm{P}_{\text {cro }}$ did not eliminate fitness costs associated with spacers that target the $g p 16$ and $g p 43$ prophage loci (Fig. 1h, i). This latter finding suggests that the fitness costs associated with these spacers are caused by leaky transcription from other promoter sequences within the prophage genome. The gp43 target is located within a gene cluster that is transcribed late during the lytic cycle of $\Phi \mathrm{NM} 1^{29}$. In staphylococcal phages with related genomic architecture, this cluster lies downstream of a regulatory region that was shown to contain a conserved transcriptional terminator ${ }^{55}$, and we identified such a region in $\Phi N M 1$ between $g p 36$ and $g p 37$. Conceivably, the magnitude of the $g p 43$ spacer's fitness cost is determined by one or more late promoters which are insulated from upstream transcription during the non-inducing conditions of lysogeny, perhaps in part due to the terminator in this regulatory region.

The potential for prophage lytic regions to be de-repressed independently of spontaneous prophage induction is not unprecedented for lambdoid temperate phages, and was previously demonstrated using a genetic reporter system ${ }^{46}$. In this previous study, the authors detected slight de-repression above background even when prophages carried a non-inducible $c I$ allele similar to that of our $\Phi \mathrm{NM} 1^{\text {ind }}$ - mutant. Leaky transcription arising in this manner could likewise be responsible for the fitness costs detected in our study. A model such as this would perhaps explain the exacerbated fitness cost observed with double lysogens where both prophages were targeted with the gp16 spacer (Fig. 3e), if we assume that transcription of each prophage is only well-coordinated in cells undergoing prophage induction ${ }^{56}$. In this scenario, if instances of leaky transcription in each prophage were poorly correlated within individual cells, the combined effect of having both targets might essentially be additive at the 
population level. Consistent with this idea, the cost associated with the $g p 16$ spacer was found to be greater in double lysogens than in single lysogens of $\Phi \mathrm{NM} 1$ and $\Phi \mathrm{NM} 4$ alone (Fig. 1c and Supplementary Fig. 3a). Examples of uncorrelated leaky expression from distinct chromosomal loci in bacteria are also not unprecedented. It was previously demonstrated that cell-cell variability becomes elevated under transcriptionally repressed conditions, even when expression from two loci with identical promoters and identical repressors is compared ${ }^{57}$. Similar singlecell approaches could be used to clarify whether expression of the two prophages used in this study is occasionally uncoupled.

Additional evidence supporting transcription-dependent chromosomal targeting as the source of the fitness costs was provided by experiments with mutant type III-A Cas nucleases. Ablation of csm3's RNase activity did not reduce fitness costs (Fig. 2b). Given its role in specific cleavage of target RNAs by type III systems ${ }^{13,58,59}$, this was perhaps expected; knockdown of prophage lytic transcripts should not be toxic to lysogenic cells per se. Meanwhile, fitness costs appeared to be completely abolished by mutation of cas 10 's palm polymerase domain (Fig. 2c), in line with past reports indicating that transcription-dependent DNA degradation by this system requires an intact palm polymerase domain ${ }^{13}$. Interestingly, mutation of csm6's HEPN RNase domain strongly reduced the cost associated with the gp16 spacer as well (Fig. 2d, e). Our previous study ${ }^{35}$ showed that Csm6's RNase activity contributes to the degradation of phage lytic transcripts, and this should not necessarily be toxic for lysogens. However, given that the specificity of Csm6's RNA knockdown effect has not been fully determined, it is possible that this activity could cause occasional off-target cleavage of essential host transcripts, and detectably impact fitness in our competition assays. Of relevance here, it was recently shown that the related HEPN domains of type VI effector RNases can mediate cleavage of nontarget transcripts, or "collateral" RNA cleavage, in the presence of target RNAs 8 . Growth defects associated with type VI targeting in vivo were accordingly proposed to result from toxic off-target cleavage of cellular transcripts ${ }^{60,61}$. More recently, binding of target RNAs by type III complexes was found to enable synthesis of a cyclic oligonucleotide that stimulates the RNase activity of Csm6 proteins, and this depended on the Cas10 subunit's intact palm polymerase domain ${ }^{62,63}$. The phenotypic similarity observed with our cas 10 and $\operatorname{csm} 6$ mutants in fitness assays (Fig. 2) might therefore be explained, at least in part, by a dominant effect of the cas 10 palm domain mutation.

The results presented in this work suggest that, among (poly) lysogenization-prone populations of $S$. aureus ${ }^{50,51}$, natural selection could favor the persistence of conditionally tolerant type III-A systems with spacers that are partially mismatched relative to their temperate phage targets. In line with this possibility, a putative conditionally tolerant isolate of $S$. aureus was recently reported to contain five mismatches in each prophage target relative to a spacer in its type III-A CRISPR-Cas system ${ }^{49}$. Conceivably there are various evolutionary routes that could lead to such co-existences between a type III-A system and one or more partially mismatched prophage targets. Although the spacers acquired upon phage infection during the adaptive stage of the CRISPR-Cas immune response are expected to match their original targets with perfect complementarity ${ }^{3}$, conditionally tolerant type III-A spacers can allow for stable lysogenization even if they are perfectly matching ${ }^{29}$. However, as our current work illustrates, the resulting conditionally tolerant (poly)lysogens can suffer fitness costs. In principle, mutations which alleviate these fitness costs could progressively accumulate in the spacer or prophage target sequence(s) after (poly)lysogeny is established. This, however, is unlikely to happen given the low frequency of spontaneous mutation in bacteria ${ }^{64,65}$. In addition, most of the cells that acquire a new spacer during phage infection will not become lysogens (depending on the phage and host, the lysogenization frequency in staphylococci varies between $10^{-3}$ and $\left.10^{-4}\right)$. We believe that these non-lysogens could provide an alternative route for the establishment of conditionally tolerant mismatched spacers with minimal fitness costs. Due to the tolerance of spacer-target mismatches that is characteristic of type III-A CRISPR-Cas immunity $29,35,53,54$, the new spacer could provide efficient defense against related temperate phages with incomplete homology with their target sequence. If infection with such phage were to result in type III-A conditional tolerance and lysogenization, the spacer will contain mismatches with the prophage target that could lead to its persistence without a fitness cost. Interestingly, in Pseudomonas aeruginosa, it was previously found that five spacer-target mismatches could enable coexistence between a type I-F system and its prophage target, although these mismatches abolished immunity to lytic infections ${ }^{43}$. Intriguingly, chromosomal targeting effects were not completely abrogated in that scenario, and were sufficient to drive a host-encoded response that inhibits group behaviors in surfacegrown populations ${ }^{66,67}$. Whether or not type III-A chromosomal targeting could give rise to a similar environment-specific phenotype remains to be investigated. Finally, it is important to note that type III-A systems have also been found to display signatures of horizontal acquisition within S. aureus genomes ${ }^{49,68,69}$. Hence, we further speculate that the genetic stability afforded by conditional tolerance in the presence of spacer-target mismatches, could be achieved through the horizontal dissemination of prophage-targeting type III-A systems into (poly)lysogenic populations harboring prophages containing mismatches with the targeting spacers. Additional investigation will be required to establish how these features of type III-A temperate phage targeting influence fitness in actively CRISPR-adapting populations, where spacer diversity is potentially much greater.

\section{Methods}

Bacterial strains and growth conditions. Unless otherwise noted, cultivation of $S$ aureus $\mathrm{RN} 4220^{37}$ or TB4 ${ }^{38}$ and their derivatives was carried out in sterile 15 or 50 $\mathrm{ml}$ conical-bottom Falcon tubes (Corning) containing Difco TSB liquid media (volume $\leq 14 \%$ tube capacity), or on sterile petri dish plates containing Difco TSA solid media. Media was supplemented with erythromycin (Erm) at $10 \mu \mathrm{g} / \mathrm{ml}$ or tetracycline (Tet) at $5 \mu \mathrm{g} / \mathrm{ml}$ only when streaking out single colonies of marked TB4 derivatives containing $\mathrm{pE} 194^{41}$ or $\mathrm{pT} 181^{42}$ plasmids, respectively, and during the construction of these strains as needed. All media was supplemented with chloramphenicol $(\mathrm{Cm})$ at $10 \mu \mathrm{g} / \mathrm{ml}$ when cultivating strains with CRISPR-Cas plasmids and/or $\mathrm{CaCl}_{2} 5 \mathrm{mM}$ if the media was intended to support phage infection and transduction. Unless otherwise noted, plates were incubated at $37^{\circ} \mathrm{C}$ for $12-18 \mathrm{~h}$ and then stored at $4{ }^{\circ} \mathrm{C}$ for up to a week. Overnight liquid cultures were inoculated from single colonies (biological replicates), grown for $12-16 \mathrm{~h}$ at $37^{\circ} \mathrm{C}$ with shaking, and then stored at $4^{\circ} \mathrm{C}$ for $3 \mathrm{~h}$ or less before briefly vortexing and subculturing at a 1:100 dilution as needed. Where applicable, subcultures were treated with Mitomycin C (AG Scientific) to a final concentration of either 1.0 or $2.0 \mu \mathrm{g} /$ $\mathrm{ml}$, or the virulent phage $\phi \mathrm{NM} 1 \gamma 6$ at a multiplicity of infection $\sim 7.5$, as indicated in the figure legends. When the overnight cultures were to be used in multi-day competition experiments, $24 \mathrm{~h}$ of growth was instead allowed, followed by immediate vortexing, mixing of aliquots, and passaging at a 1:1000 dilution. When streaking out lysogens with targeting spacers, aberrantly large colonies were presumed to have lost conditional tolerance either via genetic inactivation of CRISPR-Cas plasmids or prophage deletion, and not studied further in this work.

DNA preparation and cloning. Plasmid DNA of E. coli DH5 $\alpha$ was purified from 4 to $6 \mathrm{ml}$ overnight cultures using plasmid miniprep reagents from Qiagen, according to the manufacturer's protocol. DNA from S. aureus RN4220 or TB4 was purified similarly except that $2 \mathrm{ml}$ overnight cultures were used, and cells were treated with $10-15 \mu \mathrm{l}$ lysostaphin $\left(1 \mathrm{mg} \mathrm{ml}^{-1}\right)$ at $37^{\circ} \mathrm{C}$ for $1.5 \mathrm{~h}$ immediately after resuspension in P1 buffer. Minipreps were carried out with either Qiagen or EconoSpin columns. For PCR-based cloning procedures, DNA was amplified with Phusion polymerase (Thermo) and purified using Qiagen reagents and EconoSpin columns. When generating amplicons for Sanger sequencing, TopTaq polymerase (Qiagen) was often used for amplicons smaller than $750 \mathrm{bp}$, and DNA was not necessarily purified after the PCRs. 
Electrocompetent RN4220 cells (described previously ${ }^{29}$ ) were used for cloning in S. aureus. The pGG79 parent vector (Supplementary Table 3) is a derivative of pGG3-BsaI ${ }^{29}$ with the BsaI placeholder sequence at position two trimmed at its $3^{\prime}$ end by one base pair, and a CRISPR repeat inserted immediately downstream. This was accomplished by inverse $\mathrm{PCR}^{70}$ using primers oGG281/oGG282 (Supplementary Table 4), followed by blunt ligation. The CRISPR array was sequenced by Sanger as described previously ${ }^{29}$, and the expected plasmid size was verified via analytical digestion during subsequent manipulations. The immune functionality of this plasmid in S. aureus was later also confirmed by resistance to infection with $\phi N M 4 \gamma 4 \alpha 2$, a derivative of $\phi N M 4 \gamma 4^{24}$ possessing a target for the nes spacer in position one of pGG79's CRISPR array. Except where noted, all other CRISPR-Cas plasmids with wild-type cas genes were constructed via scarless insertion of spacers between CRISPR repeats 2 and 3 of the parent vector, pGG79. This was accomplished by restriction digesting the two oppositely oriented BsaI sites and then ligating them with an annealed oligonucleotide pair possessing compatible overhangs and the desired spacer sequence (Supplementary Tables 3 and 4). CRISPR array modifications were then verified again as described previously ${ }^{29}$. CRISPR-Cas plasmids with the $g p 16$ spacer and one or more cas gene mutations were also constructed by the annealed oligo cloning method in most cases, and checked similarly, except that mutant parent vectors were used as backbones for insertion of the spacer. The $\operatorname{csm} 3$ (D32A) mutant vector, pPS95, was constructed via 2-piece Gibson assembly of PCR products ${ }^{71}$ amplified from pGG79 using primers PS153/PS465 and PS154/PS466. The csm6 (R364A, H369A) mutant vector, pGG99, as well as the $\operatorname{csm} 3 / \mathrm{csm} 6$ double mutant vector, pGG89, were also constructed via 2piece Gibson. For pGG99, the fragment containing the CRISPR array was amplified from pGG79 using primers W852/PS566, while the rest of the backbone including the csm6 mutations was amplified from pWJ241 ${ }^{35}$ using primers PS565/W614. The same was done for pGG89, except that pWJ $242^{35}$ was used for templating the backbone with both csm 3 and csm 6 mutations. To construct the pGG167 cas 10 (D586A, D587A) palm domain mutant plasmid harboring the gp16 spacer, 2-piece Gibson was performed with a CRISPR-containing fragment amplified from pGG102 using primers W852/oGG458, and a backbone fragment amplified from pGG139 using primers oGG457/W614. The pGG139 vector contains cas 10 mutations in both the HD (H14A, D15A) and palm polymerase (D586A, D587A) domains, and was itself constructed via 2-piece Gibson of a fragment amplified from pGG79 with W852/oGG425 and a fragment amplified from pAV71 using primers oGG424/ W614. Following Gibson assembly of these vectors, the expected plasmid size was confirmed by restriction digest, and regions of interest were sequenced by Sanger using primers listed in Supplementary Table 4 (Gibson assembly junctions, CRISPR arrays, and in select cases the relevant cas nuclease active sites). The pAV71 vector was constructed via 2-piece Gibson of a fragment containing the HD domain mutation amplified from pLM546 ${ }^{72}$ with W125/PS557, and a fragment containing the palm domain mutation amplified from pWJ291 with PS556/W762. The pWJ291 vector was constructed via 2-piece Gibson of fragments amplified from pWJ30 ${ }^{29}$ using primer pairs W852/W1169 and W1170/W614. JW233 is a derivative of RN4220 with a type III-A CRISPR-Cas plasmid that targets $\phi 11$ 's $c I$-like repressor gene. This plasmid was constructed from pGG3-BsaI according to the annealed oligo cloning procedure described previously ${ }^{29}$, using oligos JW398 and JW399 for insertion of the spacer. The pAV43 plasmid was constructed by 3-piece Gibson assembly of two homology fragments amplified from $\$ N M 4 \gamma 4$ (AV205/AV202 and AV203/AV185), along with a backbone fragment amplified from pC194 (AV186/ AV204). The pE194-tyc1 plasmid was constructed by 1-piece Gibson assembly of PCR products generated with primers oGG297/oGG298. The presence of an intact assembly junction, including the $5^{\prime}$ UTR SNP in ermC, was subsequently confirmed by Sanger sequencing with primers oGG191/W235. The pGG90 plasmid was constructed by 2-piece Gibson assembly of PCR products; its integrase homology fragment was amplified from $\phi N M 1$ with primers oGG315/oGG316, while its backbone fragment including ermC homology was amplified from $\mathrm{pE}$ 194-tycl using primers oGG314/oGG317. Intact assembly junctions were verified by PCR and Sanger sequencing with primers W234/W235.

Chemically competent DH5 $\alpha$ cells were used for cloning pWJ327-derived vectors in E. coli, each constructed via 3-piece Gibson assembly of PCR products. The pGG170 plasmid contains homology to $\phi \mathrm{NM} 1$ with mutations in the $\mathrm{P}_{c r}$ promoter, while the pGG172 plasmid contains homology to $\$ \mathrm{NM} 1$ with mutations in its $c I$-like repressor. A backbone fragment was amplified from pWJ327 using primers JW809/JW810, while the homology arms were amplified from $\phi N M 1$ using primer pairs oGG467/oGG468 and oGG469/oGG470 for pGG170, or oGG471/oGG472 and oGG473/oGG474 for pGG172. The expected size of the resulting plasmids was confirmed by restriction digest, and homology arm sequences were confirmed by Sanger using primers listed in Supplementary Table 4 (W1250, oGG455, and oGG469 or W1250, oGG471, and oGG473). Unexpectedly, the plasmids failed to transform RN4220. PCR and Sanger sequencing of the region encoding elements required for replication in S. aureus at $28^{\circ} \mathrm{C}$ revealed a SNP within the temperature-sensitive repA gene, resulting in a premature stop codon. The SNP was corrected in each case to create "pGG170ts" or "pGG172ts" via 1-piece Gibson of PCR products amplified from pGG170 or pGG172 using primers oGG509/oGG510, followed by electroporation directly into RN4220 and plating at $28^{\circ} \mathrm{C}$.

Estimation of phage lysate titers. As needed, phage lysate titers were estimated by spotting serial dilutions on a lawn of sensitive bacteria to enumerate pfu, according to the procedure described previously ${ }^{29}$.
Preparation of transducing lysates. Overnight culture aliquots (100 $\mu$ l) of RN4220 or TB4 derivatives harboring a plasmid of interest were infected with "clear" mutant (lytic) derivatives of either $\phi \mathrm{NM} 1, \phi \mathrm{NM} 2, \phi \mathrm{NM} 4$, or $\phi 11$ (MOI 0.1-10) in HIA soft agar supplemented with $\mathrm{CaCl}_{2}$ at $5 \mathrm{mM}$, and incubated overnight at $37^{\circ} \mathrm{C}$ to produce a lysed lawn. Within $48 \mathrm{~h}$ of storage at $4{ }^{\circ} \mathrm{C}$, soft agar was scraped and decanted into a $50 \mathrm{ml}$ conical-bottom Falcon tube, supplemented with an additional $1.2 \mathrm{ml}$ fresh HIB, and then centrifuged at $4303 \times g$ for $8-10 \mathrm{~min}$. Filtered supernatants containing the transducing particles were stored in autoclaved $1.5 \mathrm{ml}$ tubes (Eppendorf) at $4{ }^{\circ} \mathrm{C}$. When the plasmid of interest carried a CRISPR-Cas system, care was taken to lyse its host with a suitable non-targeted phage, among the following: $\phi \mathrm{NM} 1 \gamma 6^{29}, \phi \mathrm{NM} 2 \gamma 1, \phi \mathrm{NM} 1 \gamma 6 \alpha 1, \phi \mathrm{NM} 4 \gamma 4^{24}$, or $\$ 11 \gamma 2 \beta 1$. Phages not described previously were procured via CRISPR-assisted editing as outlined below.

CRISPR-assisted editing of phages. Recombinant phages were isolated by using type III CRISPR-Cas plasmids to counter-select against different target sequences. The $\$ 11 \gamma 2 \beta 1$ phage is a mutant of $\$ 11 \gamma 2$ isolated during infection of lawns harboring the pGG102 plasmid ( $g p 16)$. After an additional round of counter selection with pGG102, the presence of a $357 \mathrm{bp}$ deletion encompassing the target site was confirmed by PCR and Sanger sequencing with primers oGG387/oGG388. $\$ 11 \gamma^{2}$ is a clear mutant of $\$ 11$ that was isolated during infection of JW233 lawns harboring a type III plasmid targeting its $c I$-like repressor gene. Following plaque reisolations, a $791 \mathrm{bp}$ deletion was confirmed by PCR and Sanger sequencing with primers JW443/JW444. The $\phi N M 1 \gamma 6 \alpha 1$ phage is a mutant of $\phi N M 1 \gamma 6$ that was similarly isolated while infecting lawns harboring the pGG100 plasmid $\left(g p 32^{\star}\right)$, although the presence of a target site deletion was not confirmed after plaque reisolations. $\phi \mathrm{NM} 2 \gamma 1$ is a clear mutant of $\phi \mathrm{NM} 2$ that was isolated during infection of lawns harboring the pGG152 type III plasmid targeting its $c I$-like repressor gene, although the presence of a target site deletion was not confirmed after plaque reisolations. For homology-directed editing of phages, we used a variation of phage editing methods described previously ${ }^{73,74}$. Briefly, phages were propagated on hosts containing homology plasmids for recombination with phage genomes, and the resultant lysates were used to infect soft agar lawns containing a suitable CRISPR-Cas plasmid that can counter-select against parental genotypes. Homologies were designed such that recombinant phages would lack a target sequence for the type III CRISPR-Cas plasmid used in counter selection. The $\phi N M 4 \gamma 4 \alpha 2$ phage was created by replacing a $40 \mathrm{bp}$ sequence containing the $g p 32$ spacer's partially matched target in $\phi \mathrm{NM} 4 \gamma 4$ with a perfectly matched target sequence for the nes spacer ${ }^{4}$. $\phi N M 4 \gamma 4$ was first propagated on lawns of RN4220 containing the homology plasmid, pAV43, and recombinants were subsequently selected during infection of lawns containing the pGG12 ${ }^{29}$ CRISPR-Cas plasmid. After an additional round of counter selection with pGG12, a single $\phi \mathrm{NM} 4 \gamma 4 \alpha 2$ plaque was isolated, and the presence of its recombinant target was confirmed by Sanger sequencing of PCR products generated with primers oGG38/oGG40. The $\phi \mathrm{NM1}$ $\mathrm{Erm}^{\mathrm{R} 2}$ phage was constructed similarly by first propagating the $\phi$ NM1-Erm ${ }^{\mathrm{R}}$ phage ${ }^{29}$ on lawns containing the pGG90 homology plasmid, which includes the tyc-1 allele SNP in the $5^{\prime}$ UTR of ermC, and subsequently selecting for recombinants lacking a nearby $182 \mathrm{bp}$ fragment that encompasses the target for the pGG91 CRISPR-Cas plasmid. The presence of the recombinant sequences in $\phi \mathrm{NM} 1$ $\mathrm{Erm}^{\mathrm{R} 2}$ was confirmed by PCR and Sanger sequencing with primers oGG192/ oGG191.

Transduction. Subcultures were grown at $37^{\circ} \mathrm{C}$ in TSB supplemented with $\mathrm{CaCl}_{2}$ at $5 \mathrm{mM}$ for $1 \mathrm{~h} 25 \mathrm{~min}\left(\sim 0.5-1.0\right.$ attenuance, $\left.D_{600} \mathrm{~nm}\right)$, and $990 \mu \mathrm{l}$ aliquots were subsequently mixed with $10 \mu \mathrm{l}$ of transducing lysate containing the plasmid of interest in autoclaved $1.5 \mathrm{ml}$ tubes (Eppendorf). After $15 \mathrm{~min}$ of growth at $37^{\circ} \mathrm{C}$, infected cultures were treated with filter-sterilized sodium citrate to a final concentration of $40 \mathrm{mM}$, and then pelleted by centrifugation at $16,100 \times g$ for 2 min with refrigeration $\left(4^{\circ} \mathrm{C}\right)$. When transducing pE194 or pT181 plasmids, an additional $1 \mathrm{~h} 45 \mathrm{~min}$ of growth was allowed immediately after treatment with citrate and prior to centrifugation. Following centrifugation, fresh TSB supplemented with sodium citrate $(40 \mathrm{mM})$ was used to resuspend and dilute pellets for plating on solid media supplemented with both sodium citrate at $20 \mathrm{mM}$ and antibiotics to select for the transductants. To avoid residual free phage, an additional re-streak was performed on solid media in the presence of citrate $(20 \mathrm{mM})$ and antibiotics to select for the strain's plasmid(s).

Phage-sensitivity assay. Throughout cloning and strain construction procedures, or as otherwise needed, clones were readily checked for sensitivity to a phage of interest according to the streak method previously performed with $\phi N M 2^{29}$, except that "clear" mutant (lytic) derivatives described above were used in place of their parental temperate phages to facilitate scoring, and chloramphenicol-supplemented TSA without added $\mathrm{CaCl}_{2}$ was usually used in place of HIA

Strain construction. Single lysogens of TB4 were obtained by mixing $100 \mu \mathrm{l}$ of a $\mathrm{TB} 4$ overnight culture with either $\phi \mathrm{NM} 1$ or $\phi \mathrm{NM} 4$ (MOI 0.1-10) in HIA soft agar supplemented with $\mathrm{CaCl}_{2}$ at $5 \mathrm{mM}$, incubating overnight at $37^{\circ} \mathrm{C}$, and restreaking from the resulting turbid lysate lawns. After an additional re-streak from single colonies, a clone was selected for each, and immunity groups were confirmed 
by assaying for sensitivity to clear mutants ( $\phi \mathrm{NM} 1 \gamma 6^{29}$ or $\left.\phi \mathrm{NM} 4 \gamma 4^{24}\right)$. Both clones were also typed via PCR using $\phi N M 1$ - or $\phi N M 4$-specific primer pairs, oGG6/ oGG7 or oGG10/oGG11, respectively. The TB4:: $\$ N M 4+\phi N M 1$ double lysogen was constructed and typed similarly, except that an overnight culture of TB4:: $\phi N M 4$ was mixed with $\phi N M 1$ in soft agar to produce the turbid lysate lawn. $\Phi \mathrm{NM1}$ prophage mutants were constructed via CRISPR-assisted genome editing as outlined below. All plasmid-containing lysogenic derivatives were subsequently generated via transduction. Plasmid-containing non-lysogens were generated either via transformation of electrocompetent TB4 cells, or via transduction. Where applicable, strains were always marked via transduction with pE194 or pT181 plasmids first, followed by delivery of the CRISPR-Cas plasmid in a subsequent round of transduction.

CRISPR-assisted genome editing of $\boldsymbol{S}$. aureus. Homology-directed allelic exchange coupled with CRISPR-Cas counter selection was performed essentially as described previously for the pWJ327 allelic replacement system ${ }^{75}$, with modifications. pWJ327-derived vectors containing homology to $\phi \mathrm{NM} 1$ and the prophage mutation of interest were used to transform electrocompetent RN4220 nonlysogens instead of RN4220:: $\Phi 12$, and a subsequent transduction step was used to transfer the plasmids from RN4220 to TB4::ФNM1. Transduction was performed with $\Phi N M 4 \gamma 4$ as described above, except that transducing lysates were raised and injected at $28^{\circ} \mathrm{C}$, with $2 \mathrm{~h} 30 \mathrm{~min}$ of growth allowed for the recipient subculture. After isolating putative co-integrants via two consecutive re-streaks at $37^{\circ} \mathrm{C}$ in the presence of chloramphenicol and verifying them with PCR using primers W1250/ oGG7 (pGG170ts) or W1250/oGG32 (pGG172ts), clones were inoculated overnight in plain media at $37^{\circ} \mathrm{C}$ instead of $28^{\circ} \mathrm{C}$. Subcultures were grown at $28^{\circ} \mathrm{C}$ in plain media supplemented with $\mathrm{CaCl}_{2}$ at $5 \mathrm{mM}$ to obtain logarithmic-phase cultures for treatment with the pWJ326 phagemid, but treated cultures were plated at $30^{\circ} \mathrm{C}$ on media containing erythromycin after $3 \mathrm{~h}$ of growth at $30^{\circ} \mathrm{C}$ rather than 1 h. An additional re-streak at $30^{\circ} \mathrm{C}$ was performed in the presence of erythromycin before clones were checked by PCR and Sanger sequencing with primers oGG455/ oGG470 (pGG170ts) or oGG471/oGG474 (pGG172ts) for the presence of the desired mutation ( $\phi \mathrm{NM} 1^{\mathrm{P} c r o}$ or $\phi \mathrm{NM} 1^{\text {ind }-}$, respectively). Clones which had also lost the integrated pWJ327 amplicon (again determined by PCR with primers W1250/oGG7 or W1250/oGG32) were inoculated overnight in plain media but grown at $42^{\circ} \mathrm{C}$ instead of $37^{\circ} \mathrm{C}$ in order to cure the strain of pWJ326. Dilutions were plated on plain media at $37^{\circ} \mathrm{C}$, and single colonies were replica-plated at $37^{\circ}$ $\mathrm{C}$ on both plain and erythromycin-supplemented media to confirm plasmid loss. After re-streaking an erythromycin-sensitive clone on plain media, a PCR amplicon spanning the entire homology region was procured with primers oGG33/oGG7 (pGG170ts) or oGG475/oGG32 (pGG172ts), and sequenced by Sanger using additional primers listed in Supplementary Table 4. The clone was also re-streaked on media supplemented with chloramphenicol as a final check for sensitivity to the antibiotic; and, checked for sensitivity to $\Phi \mathrm{NM} 1 \gamma 6$ to ensure that lysogenic immunity had not been lost. The pGG170ts- and pGG172ts-derived strains were renamed GWG7 and GWG9, respectively. TSB and TSA media were used in place of BHI throughout. The pWJ326 phagemid lysate used in this work was procured using the method described previously ${ }^{75}$.

Pairwise competition assays with marked lysogens. Aliquots of overnight cultures were mixed 1:1 by volume and then diluted for passaging and selective plating on tetracycline $(5 \mu \mathrm{g} / \mathrm{ml})$ and chloramphenicol or erythromycin $(5 \mu \mathrm{g} / \mathrm{ml})$ and chloramphenicol. Passaging and plating was repeated in this manner every 24 $\mathrm{h}$ for 6 days, and relative frequencies were calculated at each interval as the number of $\mathrm{Tet}^{\mathrm{R}}+\mathrm{Cm}^{\mathrm{R}}$ colony forming units (CFU) divided by the sum of $\mathrm{Erm}^{\mathrm{R}}+\mathrm{Cm}^{\mathrm{R}}$ CFU plus $\mathrm{Tet}^{\mathrm{R}}+\mathrm{Cm}^{\mathrm{R}}$ CFU.

\section{Enumeration of plaque-forming units from lysogenic cultures. "Spontaneous"} particle release from single lysogens was measured essentially as described previously $^{29}$, except that filtered supernatants were collected immediately after $12 \mathrm{~h}$ of growth overnight. For quantification of particles released from MMC-induced cultures, overnight cultures were grown for $15 \mathrm{~h}$ and subcultures were grown for 1 h 15 min $\left(\sim 0.3-0.6\right.$ attenuance, $\left.D_{600} \mathrm{~nm}\right)$ before treatment with MMC. Following 4 $\mathrm{h}$ of growth post treatment, subcultures were pelleted by centrifugation at $4303 \times g$ for $6 \mathrm{~min}$, and filtered supernatants were collected for serial dilution and spotting on lawns of TB4 harboring the CRISPR-Cas parent vector.

High-resolution growth curves. Overnight cultures grown for $14 \mathrm{~h}$ were subcultured essentially as described above, except that $200 \mu \mathrm{l}$ culture volumes were used in 96-well microplates, and incubation at $37^{\circ} \mathrm{C}$ with shaking was performed with an Infinite M200 PRO plate reader (TECAN) measuring attenuance $\left(D_{600 \mathrm{~nm}}\right)$ every $10 \mathrm{~min}$. In prophage induction experiments, subcultures were treated with MMC to a final concentration of $2.0 \mu \mathrm{g} / \mathrm{ml}$, following $70 \mathrm{~min}$ of growth. In phage infection experiments, subcultures were treated with $\$ \mathrm{NM} 1 \gamma 6$ at a multiplicity of infection $~ 7.5$, following $45 \mathrm{~min}$ of growth. For each overnight culture, an additional subculture was performed in parallel to monitor growth in the absence of MMC or $\phi N M 1 \gamma 6$ treatment.
Efficiency of plaquing. Assays were performed by spotting high-titer lysate serial dilutions onto both CRISPR-containing and control lawns to enumerate pfu and calculate efficiency as pfu on CRISPR-containing cells/pfu on control cells, as described previously ${ }^{29}$, except that the sensitive control lawns utilized a TB4 derivative harboring the pGG79 parent vector.

Lysogenization with ermC-marked ФNM1. Lysogenization was performed essentially as described previously ${ }^{29}$, except that overnight cultures were always grown for $14 \mathrm{~h}$, TSB broth was used in place of HIB, multiplicity of infection was $\sim 1$, and incubation times were reduced to $10 \mathrm{~min}$ on ice and $20 \mathrm{~min}$ at $37^{\circ} \mathrm{C}$. Following the $20 \mathrm{~min}$ incubation, cultures were supplemented and plated with citrate as described for the transduction protocol above, except that chloramphenicol was maintained in all media and plates were also supplemented with erythromycin $(5 \mu \mathrm{g} / \mathrm{ml})$ to quantify acquisition of the marked prophage. The $\Phi$ NM1-Erm ${ }^{\mathrm{R} 2}$ phage used for infections is a variant of $\Phi$ NM1-Erm ${ }^{\mathrm{R} 29}$ with a modified erm $C 5^{\prime}$ UTR that includes the SNP found in the tyc-1 allele, previously reported to improve constitutive expression of the cassette's gene product in $B$. subtilis $^{76}$. To estimate the concentration of total recipients harboring CRISPR-Cas systems, serial dilutions of the untreated overnight cultures were plated in the presence of chloramphenicol alone.

Batch competition of unmarked lysogens for deep sequencing. Aliquots of overnight cultures were mixed in equal proportions by volume and then diluted for passaging as described above. The remaining culture $(\sim 2 \mathrm{ml})$ was pelleted by centrifugation at $4303 \times g$ for $6 \mathrm{~min}$. Supernatants were discarded, and pellets were stored at $-20^{\circ} \mathrm{C}$ after drying for $10 \mathrm{~min}$ at benchtop. Passaging and storage of pellets was repeated in this manner every $24 \mathrm{~h}$ for 3 days.

Preparation of DNA for deep sequencing. Frozen pellets from batch competition experiments were thawed on ice for $20 \mathrm{~min}$ and then miniprepped as described above. In total, $100 \mathrm{ng}$ of miniprepped DNA from each sample was used as the template for barcoded PCRs to amplify a region of the CRISPR array that fully spans spacer position 2, using forward and reverse primers with identical barcode pairs. Barcoded PCR products were gel purified and then pooled at roughly equal proportions by normalizing to the least-concentrated NanoDrop Spectrophotometer (Thermo) reading. Library preparation was subsequently carried out on the pooled sample using a TruSeq Nano DNA LT kit (Illumina).

Deep sequencing and data analysis. Deep sequencing was performed on an Illumina MiSeq essentially as described in the manufacturer's protocol for lowcomplexity amplicon sequencing with the v3 reagent kit ( 25\% PhiX spike-in). Fastq output files were parsed using the Biopython package for python, and reads containing the expected sequences with $100 \%$ identity (either forward or reverse) were tallied for each barcode set. Relative frequency datapoints are plotted as the number of reads containing a particular barcode and the perfect or partially matching spacer of interest divided by the sum of that count plus reads containing the fully mismatched spacer with the same barcode.

Pairwise competition assays with marked non-lysogens. Aliquots of overnight cultures grown for $14 \mathrm{~h}$ were mixed 1:1 by volume and then subcultured for $45 \mathrm{~min}$, prior to withdrawing an aliquot for selective plating on tetracycline $(5 \mu \mathrm{g} / \mathrm{ml})$ and chloramphenicol or erythromycin $(5 \mu \mathrm{g} / \mathrm{ml})$ and chloramphenicol, and subsequently infecting the remaining culture with $\phi N M 1 \gamma 6$ at a multiplicity of infection $\sim 7.5$. Following $23 \mathrm{~h}$ and $15 \mathrm{~min}$ of infection at $37^{\circ} \mathrm{C}$, cultures were treated with filter-sterilized sodium citrate to a final concentration of $40 \mathrm{mM}$, diluted in similarly supplemented media, and then grown on plates containing tetracycline $(5 \mu \mathrm{g} /$ $\mathrm{ml})$ and chloramphenicol or erythromycin $(5 \mu \mathrm{g} / \mathrm{ml})$ and chloramphenicol-in each case also supplemented with sodium citrate $(20 \mathrm{mM})$. Relative frequencies were calculated at each interval as the number of $\mathrm{Tet}^{\mathrm{R}}+\mathrm{Cm}^{\mathrm{R}} \mathrm{CFU}$ divided by the sum of $\mathrm{Erm}^{\mathrm{R}}+\mathrm{Cm}^{\mathrm{R}} \mathrm{CFU}$ plus $\mathrm{Tet}^{\mathrm{R}}+\mathrm{Cm}^{\mathrm{R}} \mathrm{CFU}$.

Statistical analyses. Student's $t$ test and ANOVA analyses were performed using the stats package in R (version 3.4.0), and lme analyses were performed using the afex package in R. Statistical methods were not used to predetermine sample sizes prior to experimentation.

BLAST-assisted search for self-matching spacers in Staphylococci. The 15 fully sequenced staphylococcal genomes previously determined to contain type III-A systems ${ }^{49}$ were analyzed further for the presence of CRISPR spacers that co-occur with one or more target sequences in the same genome. To search for perfectly matching targets, a FASTA-formatted master list containing their 70 unique spacers was aligned to each genome using NCBI's online megablast tool, and results were manually screened for the presence or absence of spacer hits that matched to both a CRISPR locus and at least one additional region elsewhere in the genome. Default parameters (August 2017) were used, except that the filter for lowcomplexity regions was left unchecked. To facilitate the detection of partially mismatched spacers, alignments were repeated using NCBI's online discontinuous megablast tool. Again, default parameters were used, except that template length was set to "16", template type was set to "two templates", and the filter for low- 
complexity regions was left unchecked. In order to compile the 70-spacer master list, the previously available ${ }^{49}$ collection of 39 unique spacers from S. aureus and S. argenteus type III-A systems was expanded to include spacers from the nonaureus and non-argenteus Staphylococci in this cohort. The additional spacers were manually collected from sequences located between previously defined type III-A repeats ${ }^{49}$, except for the downstream-most spacer in each CRISPR array, which was instead defined as the sequence located between a known repeat and a degenerate repeat. The sequence of each degenerate repeat was manually inferred based on its distal location in the CRISPR array, partial homology to the nearest upstream repeat, and length ( $\sim 36 \mathrm{bp})$. Spacers identified in this manner were subsequently added to the master list, if not already present within the file.

Data availability. Relevant data supporting the findings of the study are available in this published article and its Supplementary Information files, and from the corresponding author upon request.

Received: 15 June 2017 Accepted: 11 December 2017

Published online: 04 January 2018

\section{References}

1. Marraffini, L. A. CRISPR-Cas immunity in prokaryotes. Nature 526, 55-61 (2015).

2. van Houte, S., Buckling, A. \& Westra, E. R. Evolutionary ecology of prokaryotic immune mechanisms. Microbiol. Mol. Biol. Rev. 80, 745-763 (2016).

3. Barrangou, R. et al. CRISPR provides acquired resistance against viruses in prokaryotes. Science 315, 1709-1712 (2007).

4. Marraffini, L. A. \& Sontheimer, E. J. CRISPR interference limits horizontal gene transfer in staphylococci by targeting DNA. Science 322, 1843-1845 (2008).

5. Amitai, G. \& Sorek, R. CRISPR-Cas adaptation: insights into the mechanism of action. Nat. Rev. Microbiol. 14, 67-76 (2016).

6. Heler, R., Marraffini, L. A. \& Bikard, D. Adapting to new threats: the generation of memory by CRISPR-Cas immune systems. Mol. Microbiol. 93, 1-9 (2014).

7. Charpentier, E., Richter, H., van der Oost, J. \& Wshite, M. F. Biogenesis pathways of RNA guides in archaeal and bacterial CRISPR-Cas adaptive immunity. FEMS Microbiol. Rev. 39, 428-441 (2015).

8. Shmakov, S. et al. Diversity and evolution of class 2 CRISPR-Cas systems. Nat. Rev. Microbiol. 15, 169-182 (2017).

9. van der Oost, J., Westra, E. R., Jackson, R. N. \& Wiedenheft, B. Unravelling the structural and mechanistic basis of CRISPR-Cas systems. Nat. Rev. Microbiol. 12, 479-492 (2014).

10. Makarova, K. S. et al. An updated evolutionary classification of CRISPR-Cas systems. Nat. Rev. Microbiol. 13, 722-736 (2015).

11. Jinek, M. et al. A programmable dual-RNA-guided DNA endonuclease in adaptive bacterial immunity. Science 337, 816-821 (2012).

12. Westra, E. R. et al. CRISPR immunity relies on the consecutive binding and degradation of negatively supercoiled invader DNA by Cascade and Cas3. Mol. Cell 46, 595-605 (2012).

13. Samai, P. et al. Co-transcriptional DNA and RNA cleavage during type III CRISPR-Cas immunity. Cell 161, 1164-1174 (2015).

14. Zetsche, B. et al. Cpf1 is a single RNA-guided endonuclease of a class 2 CRISPR-Cas system. Cell 163, 759-771 (2015).

15. Palmer, K. L. \& Gilmore, M. S. Multidrug-resistant enterococci lack CRISPRcas. mBio. 1, e00227-00210 (2010).

16. Marraffini, L. A. CRISPR-Cas immunity against phages: its effects on the evolution and survival of bacterial pathogens. PLoS Pathog. 9, e1003765 (2013).

17. Touchon, M., Bernheim, A. \& Rocha, E. P. Genetic and life-history traits associated with the distribution of prophages in bacteria. ISME J. 10, 2744-2754 (2016).

18. Godde, J. S. \& Bickerton, A. The repetitive DNA elements called CRISPRs and their associated genes: evidence of horizontal transfer among prokaryotes. $J$. Mol. Evol. 62, 718-729 (2006).

19. Horvath, P. et al. Comparative analysis of CRISPR loci in lactic acid bacteria genomes. Int. J. Food Microbiol. 131, 62-70 (2009).

20. Chakraborty, S. et al. Comparative network clustering of direct repeats (DRs) and cas genes confirms the possibility of the horizontal transfer of CRISPR locus among bacteria. Mol. Phylogenet. Evol. 56, 878-887 (2010).

21. Seed, K. D., Lazinski, D. W., Calderwood, S. B. \& Camilli, A. A bacteriophage encodes its own CRISPR/Cas adaptive response to evade host innate immunity. Nature 494, 489-491 (2013).

22. Brouns, S. J. et al. Small CRISPR RNAs guide antiviral defense in prokaryotes. Science 321, 960-964 (2008).

23. Bikard, D., Hatoum-Aslan, A., Mucida, D. \& Marraffini, L. A. CRISPR interference can prevent natural transformation and virulence acquisition during in vivo bacterial infection. Cell Host Microbe 12, 177-186 (2012).

24. Heler, R. et al. Cas9 specifies functional viral targets during CRISPR-Cas adaptation. Nature 519, 199-202 (2015).
25. Gudbergsdottir, S. et al. Dynamic properties of the Sulfolobus CRISPR/Cas and CRISPR/Cmr systems when challenged with vector-borne viral and plasmid genes and protospacers. Mol. Microbiol. 79, 35-49 (2011).

26. Fischer, S. et al. An archaeal immune system can detect multiple Protospacer Adjacent Motifs (PAMs) to target invader DNA. J. Biol. Chem. 287, 33351-33363 (2012).

27. Jiang, W. et al. Dealing with the evolutionary downside of CRISPR immunity: bacteria and beneficial plasmids. PLoS Genet. 9, e1003844 (2013).

28. Edgar, R. \& Qimron, U. The Escherichia coli CRISPR system protects from lambda lysogenization, lysogens, and prophage induction. J. Bacteriol. 192, 6291-6294 (2010).

29. Goldberg, G. W., Jiang, W., Bikard, D. \& Marraffini, L. A. Conditional tolerance of temperate phages via transcription-dependent CRISPR-Cas targeting. Nature 514, 633-637 (2014).

30. Vercoe, R. B. et al. Cytotoxic chromosomal targeting by CRISPR/Cas systems can reshape bacterial genomes and expel or remodel pathogenicity islands. PLoS Genet. 9, e1003454 (2013).

31. Goldberg, G. W. \& Marraffini, L. A. Resistance and tolerance to foreign elements by prokaryotic immune systems-curating the genome. Nat. Rev. Immunol. 15, 717-724 (2015).

32. Heler, R. et al. Mutations in Cas9 enhance the rate of acquisition of viral spacer sequences during the CRISPR-Cas immune response. Mol. Cell 65, 168-175 (2017).

33. Gill, S. R. et al. Insights on evolution of virulence and resistance from the complete genome analysis of an early methicillin-resistant Staphylococcus aureus strain and a biofilm-producing methicillin-resistant Staphylococcus epidermidis strain. J. Bacteriol. 187, 2426-2438 (2005).

34. Deng, L., Garrett, R. A., Shah, S. A., Peng, X. \& She, Q. A novel interference mechanism by a type IIIB CRISPR-Cmr module in Sulfolobus. Mol. Microbiol 87, 1088-1099 (2013).

35. Jiang, W., Samai, P. \& Marraffini, L. A. Degradation of phage transcripts by CRISPR-associated RNases enables type III CRISPR-Cas immunity. Cell 164, 710-721 (2016).

36. Baba, T., Bae, T., Schneewind, O., Takeuchi, F. \& Hiramatsu, K. Genome sequence of Staphylococcus aureus strain Newman and comparative analysis of staphylococcal genomes: polymorphism and evolution of two major pathogenicity islands. J. Bacteriol. 190, 300-310 (2008).

37. Nair, D. et al. Whole-genome sequencing of Staphylococcus aureus strain RN4220, a key laboratory strain used in virulence research, identifies mutations that affect not only virulence factors but also the fitness of the strain. J. Bacteriol. 193, 2332-2335 (2011).

38. Bae, T., Baba, T., Hiramatsu, K. \& Schneewind, O. Prophages of Staphylococcus aureus Newman and their contribution to virulence. Mol. Microbiol. 62, 1035-1047 (2006).

39. Horinouchi, S. \& Weisblum, B. Nucleotide sequence and functional map of pC194, a plasmid that specifies inducible chloramphenicol resistance. J. Bacteriol. 150, 815-825 (1982).

40. Holt, D. C. et al. A very early-branching Staphylococcus aureus lineage lacking the carotenoid pigment staphyloxanthin. Genome Biol. Evol. 3, 881-895 (2011).

41. Horinouchi, S. \& Weisblum, B. Nucleotide sequence and functional map of pE194, a plasmid that specifies inducible resistance to macrolide, lincosamide, and streptogramin type B antibodies. J. Bacteriol. 150, 804-814 (1982).

42. Khan, S. A. \& Novick, R. P. Complete nucleotide sequence of pT181, a tetracycline-resistance plasmid from Staphylococcus aureus. Plasmid 10, 251-259 (1983).

43. Cady, K. C., Bondy-Denomy, J., Heussler, G. E., Davidson, A. R. \& O’Toole, G A. The CRISPR/Cas adaptive immune system of Pseudomonas aeruginosa mediates resistance to naturally occurring and engineered phages. J. Bacteriol. 194, 5728-5738 (2012).

44. Nanda, A. M., Thormann, K. \& Frunzke, J. Impact of spontaneous prophage induction on the fitness of bacterial populations and host-microbe interactions J. Bacteriol. 197, 410-419 (2015).

45. Erill, I., Campoy, S. \& Barbe, J. Aeons of distress: an evolutionary perspective on the bacterial SOS response. FEMS Microbiol. Rev. 31, 637-656 (2007).

46. Livny, J. \& Friedman, D. I. Characterizing spontaneous induction of Stx encoding phages using a selectable reporter system. Mol. Microbiol. 51, 1691-1704 (2004).

47. Mo, C. Y., Birdwell, L. D. \& Kohli, R. M. Specificity determinants for autoproteolysis of LexA, a key regulator of bacterial SOS mutagenesis. Biochemistry 53, 3158-3168 (2014).

48. Johnson, A. D. et al. Lambda repressor and cro--components of an efficient molecular switch. Nature 294, 217-223 (1981).

49. Cao, L. et al. Identification and functional study of type III-A CRISPR-Cas systems in clinical isolates of Staphylococcus aureus. Int. J. Med. Microbiol. 306, 686-696 (2016).

50. Aanensen, D. M. et al. Whole-genome sequencing for routine pathogen surveillance in public health: a population snapshot of invasive Staphylococcus aureus in Europe. MBio. 7, e00444-16 (2016).

51. Goerke, C. et al. Diversity of prophages in dominant Staphylococcus aureus clonal lineages. J. Bacteriol. 191, 3462-3468 (2009). 
52. Manica, A., Zebec, Z., Steinkellner, J. \& Schleper, C. Unexpectedly broad target recognition of the CRISPR-mediated virus defence system in the archaeon Sulfolobus solfataricus. Nucleic Acids Res. 41, 10509-10517 (2013).

53. Maniv, I., Jiang, W., Bikard, D. \& Marraffini, L. A. Impact of different target sequences on type III CRISPR-Cas immunity. J. Bacteriol. 198, 941-950 (2016).

54. Pyenson, N. C., Gayvert, K., Varble, A., Elemento, O. \& Marraffini, L. A. Broad targeting specificity during bacterial type III CRISPR-Cas immunity constrains viral escape. Cell Host Microbe 22, 343-353.e343 (2017).

55. Ferrer, M. D. et al. RinA controls phage-mediated packaging and transfer of virulence genes in Gram-positive bacteria. Nucleic Acids Res. 39, 5866-5878 (2011).

56. Refardt, D. Within-host competition determines reproductive success of temperate bacteriophages. ISME J. 5, 1451-1460 (2011).

57. Elowitz, M. B., Levine, A. J., Siggia, E. D. \& Swain, P. S. Stochastic gene expression in a single cell. Science 297, 1183-1186 (2002).

58. Staals, R. H. et al. RNA targeting by the type III-A CRISPR-Cas Csm complex of Thermus thermophilus. Mol. Cell 56, 518-530 (2014).

59. Tamulaitis, G. et al. Programmable RNA shredding by the type III-A CRISPRCas system of Streptococcus thermophilus. Mol. Cell 56, 506-517 (2014).

60. Abudayyeh, O. O. et al. C2c2 is a single-component programmable RNAguided RNA-targeting CRISPR effector. Science 353, aaf5573 (2016).

61. Smargon, A. A. et al. Cas13b is a type VI-B CRISPR-associated RNA-guided RNase differentially regulated by accessory proteins Csx27 and Csx28. Mol. Cell 65, 618-630.e617 (2017)

62. Kazlauskiene, M., Tamulaitis, G., Kostiuk, G., Venclovas, C. \& Siksnys, V. Spatiotemporal control of Type III-A CRISPR-Cas immunity: coupling DNA degradation with the target RNA recognition. Mol. Cell 62, 295-306 (2016).

63. Niewoehner, O. et al. Type III CRISPR-Cas systems produce cyclic oligoadenylate second messengers. Nature 548, 543-548 (2017).

64. Drake, J. W. A constant rate of spontaneous mutation in DNA-based microbes. Proc. Natl Acad. Sci. USA 88, 7160-7164 (1991).

65. Drake, J. W., Charlesworth, B., Charlesworth, D. \& Crow, J. F. Rates of spontaneous mutation. Genetics 148, 1667-1686 (1998).

66. Cady, K. C. \& O’Toole, G. A. Non-identity-mediated CRISPR-bacteriophage interaction mediated via the Csy and Cas3 proteins. J. Bacteriol. 193, 3433-3445 (2011).

67. Heussler, G. E. et al. Clustered regularly interspaced short palindromic repeatdependent, biofilm-specific death of Pseudomonas aeruginosa mediated by increased expression of phage-related genes. MBio. 6, e00129-00115 (2015).

68. Golding, G. R. et al. Livestock-associated methicillin-resistant Staphylococcus aureus sequence type 398 in humans, Canada. Emerg. Infect. Dis. 16, 587-594 (2010).

69. Kinnevey, P. M. et al. Emergence of sequence type 779 methicillin-resistant Staphylococcus aureus harboring a novel pseudo staphylococcal cassette chromosome mec (SCCmec)-SCC-SCCCRISPR composite element in Irish hospitals. Antimicrob. Agents Chemother. 57, 524-531 (2013).

70. Moore, S. D. \& Prevelige, P. E. Jr. A P22 scaffold protein mutation increases the robustness of head assembly in the presence of excess portal protein. J. Virol. 76, 10245-10255 (2002).

71. Gibson, D. G. et al. Enzymatic assembly of DNA molecules up to several hundred kilobases. Nat. Methods 6, 343-345 (2009).

72. Hatoum-Aslan, A., Maniv, I., Samai, P. \& Marraffini, L. A. Genetic characterization of antiplasmid immunity through a type III-A CRISPR-Cas system. J. Bacteriol. 196, 310-317 (2014).

73. Kiro, R., Shitrit, D. \& Qimron, U. Efficient engineering of a bacteriophage genome using the type I-E CRISPR-Cas system. RNA Biol. 11, 42-44 (2014).

74. Martel, B. \& Moineau, S. CRISPR-Cas: an efficient tool for genome engineering of virulent bacteriophages. Nucleic Acids Res. 42, 9504-9513 (2014).

75. Modell, J. W., Jiang, W. \& Marraffini, L. A. CRISPR-Cas systems exploit viral DNA injection to establish and maintain adaptive immunity. Nature 544, 101-104 (2017).
76. Gryczan, T. J., Grandi, G., Hahn, J., Grandi, R. \& Dubnau, D. Conformational alteration of mRNA structure and the posttranscriptional regulation of erythromycin-induced drug resistance. Nucleic Acids Res. 8, 6081-6097 (1980).

77. Iandolo, J. J. et al. Comparative analysis of the genomes of the temperate bacteriophages phi 11, phi 12 and phi 13 of Staphylococcus aureus 8325. Gene 289, 109-118 (2002).

78. Ganguly, T. et al. Physicochemical properties and distinct DNA binding capacity of the repressor of temperate Staphylococcus aureus phage phil1. FEBS J. 276, 1975-1985 (2009).

79. Quiles-Puchalt, N. et al. Staphylococcal pathogenicity island DNA packaging system involving cos-site packaging and phage-encoded $\mathrm{HNH}$ endonucleases. Proc. Natl Acad. Sci. USA 111, 6016-6021 (2014).

\section{Acknowledgements}

We are grateful to Philip M. Nussenzweig, Robert Heler, and Pascal Maguin for additional advice on deep sequencing of low-complexity amplicon pools. We are also grateful to Robert Heler for instructions on extracting selection coefficient values from fitness data. We would like to thank Seth Darst for advice on promoter inactivation, as well as Charlie Mo for advice on active site mutations in proteins of the LexA superfamily. Finally, we would like to thank The Rockefeller University Genomics Resource Center for deep sequencing library preparation. L.A.M. is supported by the Burroughs Wellcome Fund PATH Award, an NIH Director's Pioneer Award (DP1GM128184-01) and a HHMI-Simons Faculty Scholar Award.

\section{Author contributions}

G.W.G. and L.A.M. designed experiments. G.W.G. provided the initial conception of the study and conducted the experiments. E.A.M. advised on statistical reporting and carried out statistical tests. A.V., J.W.M., P.S. and W.J. contributed reagents and technical expertise. G.W.G. and L.A.M. wrote the paper.

\section{Additional information}

Supplementary Information accompanies this paper at https://doi.org/10.1038/s41467 017-02557-2.

Competing interests: The authors declare no competing financial interests.

Reprints and permission information is available online at http://npg.nature.com/ reprintsandpermissions/

Publisher's note: Springer Nature remains neutral with regard to jurisdictional claims in published maps and institutional affiliations.

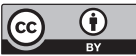

Open Access This article is licensed under a Creative Commons Attribution 4.0 International License, which permits use, sharing, adaptation, distribution and reproduction in any medium or format, as long as you give appropriate credit to the original author(s) and the source, provide a link to the Creative Commons license, and indicate if changes were made. The images or other third party material in this article are included in the article's Creative Commons license, unless indicated otherwise in a credit line to the material. If material is not included in the article's Creative Commons license and your intended use is not permitted by statutory regulation or exceeds the permitted use, you will need to obtain permission directly from the copyright holder. To view a copy of this license, visit http://creativecommons.org/ licenses/by/4.0/.

(c) The Author(s) 2017 